Article

\title{
Combining Ability of Extra-Early Maize Inbreds Derived from a Cross between Maize and Zea diploperennis and Hybrid Performance under Contrasting Environments
}

\author{
Isaac K. Amegbor ${ }^{1,2,3}{ }^{(0}$, Baffour Badu-Apraku $4, *\left(\mathbb{C}\right.$, Gloria B. Adu ${ }^{2}$, \\ Joseph Adjebeng-Danquah ${ }^{2}(1)$ and Johnson Toyinbo ${ }^{4}$ \\ 1 Department of Agronomy, Pan African University, Institute of Life and Earth Sciences (Including Health \& \\ Agriculture), University of Ibadan, Ibadan 200284, Nigeria; isaacamegbor@gmail.com \\ 2 Council for Scientific and Industrial Research (CSIR)-Savanna Agricultural Research Institute, \\ Tamale 00233, Ghana; gloriaboakyewaa@yahoo.com (G.B.A.); barchus2003@yahoo.com (J.A.-D.) \\ 3 Department of Plant Breeding, University of the Free State, Bloemfontein 9301, South Africa \\ 4 Maize Improvement Unit, International Institute of Tropical Agriculture, IITA-HQ Ibadan, PMB 5320, \\ Oyo Road, Ibadan 200284, Nigeria; o.toyinbo@cgiar.org \\ * Correspondence: b.badu-apraku@cgiar.org; Tel.: +234-810-848-2590
}

Received: 1 July 2020; Accepted: 22 July 2020; Published: 24 July 2020

check for updates

\begin{abstract}
Knowledge of the genetic mechanisms conditioning drought tolerance in maize is crucial to the success of hybrid breeding programs aimed at developing high-yielding cultivars under drought. The objectives of this study were to determine the combining ability of extra-early inbreds, compute the heritability of measured traits, assess the performance of inbreds in hybrid combinations and investigate the associations among traits under drought and optimal conditions. A total of 252 hybrids generated by crossing 63 inbreds to four testers, along with four commercial hybrid checks, were evaluated for 2 years under drought and rainfed conditions. General combining ability (GCA) and specific combining ability (SCA) for the traits were significant. A total of $57.1 \%$ and $53.4 \%$ of the genotypic sum of squares were attributable to GCA effects for grain yield under managed drought and rainfed conditions, respectively. Hybrids TZdEEI $91 \times$ TZEEI 21 and TZdEEI $55 \times$ TZEEI 13 out-yielded the best checks under drought and optimal conditions by $49.13 \%$ and $39.05 \%$, respectively. The most promising hybrids with consistently high grain yield under drought and rainfed conditions, were TZdEEI $54 \times$ TZEEI 13, TZdEEI $91 \times$ TZEEI 21 and TZdEEI $55 \times$ TZEEI 21 and should be further evaluated for possible commercial production in sub-Saharan Africa.
\end{abstract}

Keywords: drought; general combining ability; heritability; specific combining ability; variance components; Zea diploperennis; Zea mays

\section{Introduction}

Maize (Zea mays L.) is ranked among the top three most widely cultivated cereal crops globally, with a total production of 114.75 million tons in 2019 and a projected increase in production of $6.47 \%$ in 2020 [1]. In most parts of Africa, maize serves as an important staple cereal crop and is utilized in preparing a variety of local dishes and as feed for animals. Despite the enormous potential and crucial role that maize plays in sub-Saharan Africa (SSA), its production and average yield per hectare are low because of recurring droughts during the cropping season. About $15 \%$ of the annual yield loss in SSA has been attributed to drought stress [2]. Edmeades et al. [3] and Lafitte et al. [4] also reported about $17 \%$ yield loss attributable to drought stress, while drought stress in southern Africa reportedly causes 
as much as $60 \%$ yield loss $[5,6]$. Reduction in maize grain yield attributable to inadequate moisture depends on the developmental stage of the crop at which the drought occurs, and on the intensity and duration of the drought $[7,8]$.

Water requirements for maize production differ during the plant developmental stages, with a total of $250 \mathrm{~L}$ of water required per plant during the growing season $[9,10]$. The peak period of water demand during the growth cycle of the maize plant is two weeks before and after pollination $[10,11]$. Even though water is essential for the maize plant at all developmental stages, the plant is most sensitive to inadequate moisture during the flowering period, resulting in delayed silking and an increased anthesis-silking interval (ASI) $[8,12,13]$. The prolonged ASI results in poor kernel set and, consequently, reduced grain yield [14-16]. It has been established that drought stress has adverse effects on plant height, leaf area and root growth [17].

Studies have revealed that genetic enhancement of maize for drought tolerance could result in genetic gains [3,18]. Edmeades et al. [19] further pointed out that the deployment of genotypes with drought-tolerance genes is an important strategy to stabilize maize production in areas with recurrent drought. However, it is essential that agronomic practices that maximize water availability to the plant be encouraged to close the gap between potential and realized yield under water stress $[19,20]$. Therefore, genotypes with enhanced tolerance to drought could serve as invaluable germplasm resources in environments with erratic occurrence of varying intensities of drought [21]. Drought-tolerant maize varieties offer the most economic and sustainable opportunity to stabilize maize yields $[8,22]$. Therefore, an important strategy to increase maize production and productivity in SSA is to breed for drought-tolerant genotypes for resource-poor farmers.

It is of utmost importance for breeding programs to determine the general combining ability of inbred lines to be used as parents in hybrid combinations and to obtain information on specific combining ability (SCA) and heterotic patterns. Therefore, combining ability studies of inbred lines are routinely carried out to identify parental lines that could be used in developing productive hybrids [23]. Such studies are also essential in plant breeding programs for assessing the superiority of parental lines in hybrid combinations [8,24]. Results of combining ability studies indicate the predominance of SCA over GCA effects for grain yield, anthesis-silking interval, days to silking, plant height, plant and ear aspects, root lodging and ears per plant under drought stress conditions. For example, investigators have reported non-additive gene action for grain yield under drought stress to be more important than the additive gene action $[25,26]$. Contrarily, other researchers [27-29] have reported the preponderance of additive gene action for grain yield and other traits under drought stress conditions. The contrasting results may be attributed to the sources and genetic background of the inbred lines used for the different studies, the intensity of drought-stress conditions and the influence of environmental conditions, such as soil and climate.

Knowledge and understanding of the pattern of gene action governing the inheritance of traits are vital in planning effective and efficient gene-deployment schemes in a drought-tolerance deployment program. It is therefore essential to assess the combining abilities (GCA and SCA) for grain yield and other agronomic traits of the extra-early maturing maize inbred lines extracted from diverse germplasm sources in west and central Africa (WCA) so that they could be successfully used to develop hybrids with superior grain yield under contrasting environmental conditions.

The classification of inbreds into appropriate heterotic groups determines the potential usefulness of inbreds in a hybrid program. This is because it allows a better understanding of the genetic relationships among the inbreds and facilitates their effective utilization in a maize breeding program for the development of synthetic varieties, hybrids, and heterotic populations. Reports on the heterotic patterns and gene action conditioning grain yield of extra-early maize inbreds under drought stress are limited. While information is available on the heterotic patterns of the International Institute of Tropical Agriculture (IITA)'s late and intermediate maize genotypes [21,30,31], only limited information is available on the heterotic patterns and gene action modulating the inheritance of grain yield and secondary traits, such as ears per plant and stay green characteristic of the IITA's extra-early inbreds 
under drought conditions. It is therefore essential to assess the combining abilities for grain yield and other agronomic traits of the extra-early maturing inbred lines extracted from diverse germplasm sources in WCA so that they could be successfully used to develop hybrids with superior grain yield under contrasting environmental conditions.

The combining abilities of maize inbred lines used in developing superior hybrids can be determined through various mating schemes, including the diallel mating design, North Carolina Design (NCD) II and the line $\times$ tester crosses. However, when considering a large number of inbred lines for combining ability studies, the line $\times$ tester mating design becomes more appropriate and the mating design of choice, as it reduces the number of hybrids to be tested but provides essential genetic information on the germplasm tested.

A wild relative of maize, Zea diploperennis, containing valuable genes for tolerance to biotic and abiotic stresses, is of immense interest [2]. A large number of extra-early-maturing white endosperm maize inbred lines have been developed from crosses between TZEE-W Pop DT STR, an extra-early Striga-resistant and drought-tolerant white endosperm population and Zea diploperennis. However, limited information is available on the combining ability, heritability and performance of the extra-early maturing white maize inbreds in hybrid combinations under drought and optimal growing conditions. The objectives of this study were to (i) determine the combining ability for grain yield and other agronomic traits of the extra-early inbreds derived from the TZEE-W Pop DT STR $\times$ Zea diploperennis crosses, (ii) compute the broad sense and narrow sense heritabilities of grain yield and other agronomic traits, (iii) examine the performance of the inbreds in hybrid combinations and (iv) investigate the associations among measured traits under drought and optimal growing conditions.

\section{Materials and Methods}

\subsection{Development of Germplasm}

Sixty-three inbreds, selected from a panel of extra-early white endosperm inbreds from the IITA-Maize Improvement Program (IITA-MIP), were used in this study. The lines were derived from crosses between the normal endosperm white extra-early maize population, TZEE-W $\mathrm{P}_{\mathrm{OP}}$ STR $\mathrm{C}_{4}$ and four IITA's intermediate-maturing inbreds, TZSTRI 104, TZSTRI 105, TZSTRI 107, and TZSTRI 108, carrying genes for Striga resistance and drought tolerance introgressed from Z. diploperennis [8]. The $F_{1}$ hybrids were backcrossed to the extra-early population to obtain the $\mathrm{BC}_{1} \mathrm{~F}_{1}$ crosses to recover extra-earliness. This was followed by two backcrosses to the population during the growing season of 2009 to recover extra-earliness. The $\mathrm{BC}_{1} \mathrm{~S}_{1}$ families were evaluated under Striga infestation at Abuja and Mokwa in 2010 and the best families were introgressed into the extra-early population. Furthermore, the $\mathrm{BC}_{1} \mathrm{~S}_{1}$ of the extra-early population was planted during the 2010 major growing season in the IITA breeding nursery in Ibadan and inbred development was initiated. The $\mathrm{BC}_{1} \mathrm{~S}_{1}$ of the extra-early population was selfed for advancement to the $\mathrm{BC}_{1} \mathrm{~S}_{2}$ stage. Subsequently, $\mathrm{BC}_{1} \mathrm{~S}_{2}$ families of the population were advanced to the $\mathrm{BC}_{1} \mathrm{~S}_{6}$ stage in 2012 through repeated self-pollination. From this program, about 100 extra-early and 200 outstanding drought and/or Striga-resistant extra-early $\mathrm{S}_{6}$ inbreds were identified. The S6 inbreds were evaluated under Striga infestation during the growing season of 2012 and screened for drought tolerance under drought stress at Ikenne during the 2012/2013 dry season and heat stress at Kadawa during the dry season of 2013. Based on the results of the studies, a panel of sixty-three inbred lines were selected for the present study. The selected 63 lines with tolerance to drought, combined heat and drought stress as well as resistance to Striga were crossed to four elite testers, TZEEI 13, TZEEI 14, TZEEI 21 and TZEEI 29, to generate the 252 single-cross hybrids used in the present study. Four commercial hybrids from the IITA-MIP were included in the study as local checks.

Two sets of experiments were conducted using the 256 hybrids: (1) under managed-drought stress and (2) under optimal conditions. The design for each experiment was a $16 \times 16$ alpha lattice with two replications. Each experimental unit consisted of single-row plots, each row $3 \mathrm{~m}$ long, with an inter 
row spacing of $0.75 \mathrm{~m}$ and an intra-row spacing of $0.40 \mathrm{~m}$ for both experiments. Three seeds were sown per hill and seedlings were thinned to two per hill at two weeks after germination, yielding a target plant density of $66,666 \mathrm{ha}^{-1}$.

The managed drought experiment was conducted during the 2013/2014 and 2014/2015 dry seasons at Ikenne (lat. $6^{\circ} 87^{\prime} \mathrm{N}$, long. $3^{\circ} 7^{\prime} \mathrm{E} ; 1500 \mathrm{~mm}$ annual rainfall). The 2013/2014 and 2014/2015 drought experiments were planted during the last week of November and harvested during the second week of March. Each week, about $17 \mathrm{~mm}$ of water was applied to plants in the drought experiments using a sprinkler irrigation system resulting in a total of $51 \mathrm{~mm}$ of water during the entire cropping season. Drought stress was achieved by suspending irrigation between three weeks after planting (WAP) and physiological maturity to ensure that the plants depended completely on stored water in the soil and in the plant tissue for growth and development. The day temperature each year during the managed drought experiment period ranged from $32{ }^{\circ} \mathrm{C}$ in November to $36{ }^{\circ} \mathrm{C}$ in February. Contrarily, night temperature during the experimental period ranged from about 20 to $25^{\circ} \mathrm{C}$ at Ikenne. For the optimal trials, hybrids were evaluated at Ikenne during the rainy season and at Bagauda, a terminal drought-prone location, which was considered a rainfed location during the 2014 cropping season because there was no terminal drought, as it rained throughout the growing season. At Bagauda, the day temperature each year varied between $29^{\circ} \mathrm{C}$ in July and $32{ }^{\circ} \mathrm{C}$ in October while night temperature was between 21 and $23^{\circ} \mathrm{C}$.

For the managed-drought experiment, $60 \mathrm{~kg} \mathrm{ha}^{-1}$ each of N, P and K (15-15-15) was applied at planting. Top-dressing was done at $2 \mathrm{WAP}$ using urea at the rate of $60 \mathrm{~kg} \mathrm{ha}^{-1}$. In contrast, basal and top-dressing fertilizer applications were carried out at 2 and 5 WAP under rainfed conditions, as reported earlier for the managed-drought experiment. The experiments were kept weed-free using pre- (premextra) and post-emergence (gramoxone) herbicides, each at $5 \mathrm{~L} /$ ha and subsequently supplemented with manual weeding.

\subsection{Data Collection}

Observations were made on days to $50 \%$ silking (DS) as the number of days when $50 \%$ of the plants had emerged silks, while days to $50 \%$ anthesis (DA) represented the number of days when $50 \%$ of plants had shed pollen. The anthesis-silking interval (ASI) was determined as the difference between DA and DS. Other measured traits were plant height (PLHT) and ear height (EHT), measured as the distance in centimeters between the base of the plant and the first tassel branch and the top ear, respectively. Ears per plant (EPP) was obtained by dividing the number of ears harvested by number of plants at harvest. Plant aspect (PASP) was rated on a scale of $1-9$, where $1=$ excellent and $9=$ poor; and ear aspect (EASP) was recorded on a scale of $1-9$, where $1=$ clean, uniform, large, and well-filled ears and $9=$ ears with undesirable features, such as diseased, small ears, and ears with poorly filled grains. Stay green characteristic or leaf death score (LD) was determined under drought-stress conditions at 70 DAP on a scale of 1 to 9 , where $1=$ almost all leaves green and $9=$ virtually all leaves dead, as described by Amegbor et al. [2]. Harvested ears from the managed-drought trials were shelled and grain yield $\left(\mathrm{kg} \mathrm{ha}^{-1}\right)$ was determined using the shelled grain weight. Grain yield $\left(\mathrm{kg} \mathrm{ha}^{-1}\right)$ of the rainfed experiment was computed on the basis of the field weight, assuming a shelling percentage of 80 at $15 \%$ moisture content.

\subsection{Statistical Analysis}

Analyses of variance (ANOVA) were conducted separately for the drought experiment and that under optimal conditions based on plot means for the measured traits using PROC GLM in SAS version 9.3 [32]. In the analysis, locations, replications, and blocks were considered random effects, whereas entries were considered fixed effects. The genetic estimates were computed using Analysis of Genetic Designs (AGD-R version 3.0) and the Line $\times$ Tester R program [33]. Broadsense heritability $\left(\mathrm{H}^{2}\right)$ and 
narrow sense heritability $\left(\mathrm{h}^{2}\right)$ estimates of the measured traits under each management condition were computed following the methods of Singh et al. [34]:

$$
\mathrm{H}^{2}=\sigma_{\mathrm{g}}^{2} / \sigma_{\mathrm{p}}^{2}
$$

where $\sigma^{2} \mathrm{~g}=$ genotypic variance; and $\sigma^{2} \mathrm{p}=$ phenotypic variance. The $\sigma^{2} \mathrm{p}$ was computed as follows:

$$
\sigma_{p}^{2}=\sigma_{g}^{2}+\sigma_{g e}^{2} / e+\sigma^{2}{ }_{e} / r e
$$

where $\sigma^{2}$ is genotypic variance, $\sigma^{2}$ ge is genotype $\times$ environment, $r$ is number of replications, and e is number of environments under drought and optimal conditions. The standard errors of the heritability estimates of the measured traits under drought stress were computed to provide a measure of the precision of the estimates [34]. Narrow sense heritability $\left(h^{2}\right)$ was computed as follows:

$$
h^{2}=\sigma_{a}^{2} / \sigma^{2} p
$$

where $\sigma^{2}{ }_{\mathrm{a}}=$ additive genetic variance.

The superior hybrids under drought and optimal conditions were identified using the multiple trait base index (MI) proposed by Badu-Apraku et al. [18]. The index integrated grain yield and other important traits and was computed as follows:

$$
\mathrm{MI}=(2 \times \mathrm{YLD})+\mathrm{EPP}-\mathrm{EASP}-\mathrm{PASP}-\mathrm{ASI}-\mathrm{LD}
$$

where YLD = grain yield, EPP = number of ears per plant, EASP = ear aspect, PASP = plant aspect, ASI $=$ anthesis silking interval under drought and $\mathrm{LD}=$ leaf death score under drought.

Yield reduction attributable to drought stress was computed using the formula:

$$
\begin{gathered}
\text { Yield reduction }(\mathrm{YR} ; \%)=[(\text { yield under optimal conditions }- \text { yield under drought }) / \\
\text { (yield under optimal conditions })] \times 100
\end{gathered}
$$

The measured traits used in computing the base index for identification of superior hybrids under drought and optimal environments were standardized, with a mean of zero and standard deviation of 1 , to minimize the effects of different scales. Therefore, a positive index value indicated tolerance to drought, whereas a negative value indicated susceptibility to drought.

The variation among hybrids was partitioned into variation attributable to lines, testers and line $x$ tester interactions. The relative importance of general combining ability (GCA) and specific combining ability (SCA) was determined as the proportion of the genotypic sum of squares attributable to GCA and SCA [35]. If the ratio of the sum of squares attributable to GCA was $>1$, then the predictability of a specific hybrid's performance for the trait could be made on the basis of GCA; and if the ratio was $<1$, then the opposite was true [36]. Furthermore, GCA and SCA effects as well as their standard errors were computed for grain yield and other measured traits under the research environments using SAS version 9.3 [32]. The GCA effect of each female line was determined on the basis of its performance in $F_{1}$ hybrid combinations across all testers, whereas the GCA effect of a tester (male) was based on its performance in $F_{1}$ hybrid combinations across all female lines. GCA and SCA effects were determined for each trait under each research environment. The general linear model for line $\times$ tester mating design is as follows:

$$
\mathrm{Y}_{\mathrm{ijkl}}=\mu+\mathrm{a}_{1}+\mathrm{b}_{\mathrm{kl}}+\mathrm{v}_{\mathrm{ij}}+(\mathrm{av})_{\mathrm{ijl}}+\varepsilon_{\mathrm{ijk}}
$$

where $Y_{\mathrm{ijkl}}=$ observed value from each experimental unit; $\mu$ = population mean; $\mathrm{a}_{1}=$ location effect; $b_{k l}=$ block or replication effect within locations; $v_{i j}=F_{1}$ hybrid effect $=g_{i}+g_{j}+s_{i j}$, where $g_{i}=$ general combining ability (GCA) for the ith parental line; $g_{j}=$ GCA effects of $j$ th tester; $s_{i j}=$ specific combining ability (SCA) for the $i_{j 1} F_{1}$ hybrid, whereas $(a v)_{i j l}=$ interaction effect between $i_{j l} F_{1}$ hybrid and lth location; and $\varepsilon_{\mathrm{ijkl}}=$ residual effect. 


\section{Results}

3.1. Analysis of Variance and Combining Ability for Grain Yield and Other Traits of Extra-Early White Hybrids under Drought and Optimal Conditions

The analysis of variance (ANOVA) of the extra-early hybrids assessed under managed-drought stress and optimal conditions revealed significant $(p<0.001)$ hybrid $(\mathrm{G})$, environment $(\mathrm{E})$ and $\mathrm{G} \times \mathrm{E}$ interaction (GEI) mean squares for grain yield and most of the measured secondary traits (Table 1). Partitioning of the genotypic mean squares into GCA and SCA components revealed that both the GCA and SCA mean squares were significant for grain yield and for most of the measured traits under each test condition, except for the GCA of testers for grain yield under optimal growing conditions. The significant variation observed for grain yield under the managed-drought stress and non-stress environments showed that there was large genetic variability among the hybrids for grain yield and thus selection could be made from the present inbred lines and hybrids developed from Zea diploperennis to combat drought stress.

In the present study, the GCA (GCA-line + GCA-tester) variance was higher than the variance for SCA of hybrids for grain yield, DS, DA, PLHT, EASP and PASP, whereas the SCA variance of hybrids were more important for ASI, EHT, EPP and LD under drought conditions. Under optimal growing conditions, GCA variance for grain yield, DA, DS, PLHT and EHT was greater than the SCA variance, whereas the SCA variance for ASI, PASP, EASP and EPP was greater than the GCA variance.

\subsection{Performance of the Single Cross Hybrids under Managed Drought and Optimal Growing Conditions}

Under drought conditions, grain yield of the 15 best- and 10 worst-performing hybrids selected using the base index ranged from $1229 \mathrm{~kg} \mathrm{ha}^{-1}$ for TZdEEI $90 \times$ TZEEI 13 to $4480 \mathrm{~kg} \mathrm{ha}^{-1}$ for TZdEEI 91 $\times$ TZEE 14, with an average grain yield of $2539 \mathrm{~kg} \mathrm{ha}^{-1}$ (Table 2). In contrast, under optimal-growing conditions, grain yield ranged from $2219 \mathrm{~kg} \mathrm{ha}^{-1}$ for TZdEEI $107 \times$ TZEEI 21 to $8136 \mathrm{~kg} \mathrm{ha}^{-1}$ for TZdEEI $55 \times$ TZEEI 13, with a mean of $5212 \mathrm{~kg} \mathrm{ha}^{-1}$. Grain yield reduction under drought stress compared with that under optimal conditions ranged from $3.10 \%$ to $75.17 \%$ for the hybrids. Under drought conditions, the best check [(TZEEI $29 \times$ TZEEI 21) $\times($ TZEEI $14 \times$ TZEEI 37)], which is a double-cross hybrid, produced $3004 \mathrm{~kg} \mathrm{ha}^{-1}$ of grain yield, whereas the best drought-tolerant hybrid (TZdEEI 91 $\times$ TZEEI 21) identified in the present study produced $49.13 \%$ more grain yield than the best check (Table 3). Under the optimal conditions, hybrid TZdEEI $55 \times$ TZEEI 13 produced 39.05\% more grain yield than the best commercial check TZEEI $21 \times$ TZEEI 29. The significant variation observed for grain yield under drought stress further revealed the differential levels of drought tolerance among the hybrids in this study. 
Table 1. Mean squares of general combining ability (GCA) and specific combining ability (SCA) of grain yield and other agronomic traits of extra-early maturing maize hybrids evaluated under induced drought at Ikenne during the 2013 and 2014 dry seasons and under optimal growing conditions at Bagauda and Ikenne during the 2014 rainy season.

\begin{tabular}{|c|c|c|c|c|c|c|c|c|c|c|c|}
\hline Source of Variation & DF & GY $\left(\mathrm{tha}^{-1}\right)$ & DA & DS & ASI & PLHT (cm) & EHT $(\mathrm{cm})$ & EPP & PASP & EASP & LD \\
\hline \multicolumn{12}{|l|}{ Drought } \\
\hline SITE & 1 & $55,948,398.5^{* *}$ & $1150.4^{* *}$ & $1156.5^{* *}$ & $26.87 * *$ & $83,207.8^{* *}$ & $58,128.1 * *$ & $2.72 * *$ & $6.51 * *$ & $31.03^{* *}$ & $148.56^{* *}$ \\
\hline GENOTYPES & 255 & $1,020,064.9^{* *}$ & $5.4^{* *}$ & $8.2^{* *}$ & $1.73^{* *}$ & $270.1 * *$ & 100.8 ** & $0.02 * *$ & $1.14^{* *}$ & $1.33^{* *}$ & 0.78 ** \\
\hline GCA $_{\text {TESTER }}$ & 3 & $5,610,191.9 * *$ & $2.2 \mathrm{~ns}$ & $14.3^{* *}$ & $5.02 * *$ & $200.6 \mathrm{~ns}$ & $760.7^{* *}$ & $0.09^{* *}$ & $11.25^{* *}$ & $9.90 * *$ & $12.64^{* *}$ \\
\hline $\mathrm{SCA}_{\text {LINE } \times \text { TESTER }}$ & 186 & $590,103.1 * *$ & $3.3^{* *}$ & $4.70^{* *}$ & $1.25 *$ & $122.9 \mathrm{~ns}$ & $68.1 \mathrm{~ns}$ & $0.02 * *$ & $0.56 \mathrm{~ns}$ & $0.82 * *$ & $0.58^{* *}$ \\
\hline SITE $\times$ GENOTYPES & 255 & $530,535.3 *$ & $2.6 \mathrm{~ns}$ & $4.3 \mathrm{~ns}$ & $1.14 \mathrm{~ns}$ & 155.4 * & $74.71 \mathrm{~ns}$ & $0.02 *$ & $0.71 *$ & $0.74 *$ & $0.52 \mathrm{~ns}$ \\
\hline SITE $\times$ GCA $_{\text {LINE }}$ & 62 & $705,295.9^{* *}$ & $2.9 \mathrm{~ns}$ & $5.4^{*}$ & $1.31 \mathrm{~ns}$ & $190.1^{* *}$ & $76.4 \mathrm{~ns}$ & $0.02 * *$ & $0.74 \mathrm{~ns}$ & $0.89^{* *}$ & $0.49 \mathrm{~ns}$ \\
\hline SITE $\times \mathrm{GCA}_{\text {TESTER }}$ & 3 & $4355,276.3$ * & $1.7 \mathrm{~ns}$ & $4.5 \mathrm{~ns}$ & $3.58^{* *}$ & $408.1^{*}$ & $109.7 \mathrm{~ns}$ & $0.01 \mathrm{~ns}$ & $1.68^{*}$ & $5.40 * *$ & $1.34 *$ \\
\hline SITE $\times$ SCA & 186 & $410,592.4 \mathrm{~ns}$ & $2.5 \mathrm{~ns}$ & $3.8 \mathrm{~ns}$ & $1.04 \mathrm{~ns}$ & $139.7 \mathrm{~ns}$ & $73.6 \mathrm{~ns}$ & $0.01 \mathrm{~ns}$ & $0.68 \mathrm{~ns}$ & $0.62 \mathrm{~ns}$ & $0.52 \mathrm{~ns}$ \\
\hline RESIDUALS & 442 & $435,017.5$ & 2.4 & 3.4 & 0.99 & 125.3 & 66.5 & 0.01 & 0.59 & 0.59 & 0.46 \\
\hline \%GCA SS & & 57.1 & 55.2 & 57.5 & 46.5 & 66.3 & 49.9 & 38.0 & 63.3 & 54.2 & 45.2 \\
\hline$\%$ SCA SS & & 42.9 & 44.8 & 42.5 & 53.5 & 33.7 & 50.1 & 62.0 & 36.7 & 45.8 & 54.8 \\
\hline \multicolumn{12}{|l|}{$\begin{array}{c}\text { Optimal } \\
\text { Environment }\end{array}$} \\
\hline SITE & 1 & $61,272,987.1^{* *}$ & $43.2^{* *}$ & $53.0^{* *}$ & $634.91 * *$ & $12,066.3^{* *}$ & $3237.4 * *$ & $1.09 * *$ & $0.22 \mathrm{~ns}$ & $0.2 \mathrm{~ns}$ & - \\
\hline GENOTYPES & 255 & $2,610,900.8 * *$ & $5.4^{* *}$ & $5.2 * *$ & $0.60 \mathrm{~ns}$ & $434.7 * *$ & $159.5^{* *}$ & $0.02 * *$ & $0.17^{* *}$ & $0.5^{* *}$ & - \\
\hline $\mathrm{GCA}_{\mathrm{LINE}}$ & 62 & $5,557,336.0 * *$ & $10.1^{* *}$ & $9.4^{* *}$ & $0.62 \mathrm{~ns}$ & $1073.9^{* *}$ & $332.8^{* *}$ & $0.03 * *$ & $0.29 * *$ & $0.4^{* *}$ & - \\
\hline $\mathrm{GCA}_{\text {TESTER }}$ & 3 & $1,808,244.5 \mathrm{~ns}$ & $45.2 * *$ & $40.8^{* *}$ & $1.03 \mathrm{~ns}$ & $4149.7 * *$ & $781.6^{* *}$ & $0.11 * *$ & 0.40 ** & $2.1 * *$ & - \\
\hline SITE $\times$ GENOTYPES & 255 & $1,094,928.5^{* *}$ & $2.8^{* *}$ & $2.7^{*}$ & $0.58 \mathrm{~ns}$ & $161.3^{* *}$ & $77.4 \mathrm{~ns}$ & $0.02 \mathrm{~ns}$ & 0.11 ** & $0.2^{* *}$ & - \\
\hline SITE $\times \mathrm{GCA}_{\mathrm{LINE}}$ & 62 & $1,392,071.9 * *$ & $3.7^{* *}$ & $3.3^{*}$ & $0.56 \mathrm{~ns}$ & $212.1^{* *}$ & $115.0^{* *}$ & $0.02 \mathrm{~ns}$ & $0.17^{* *}$ & $0.2 * *$ & - \\
\hline SITE $\times$ GCA $_{\text {TESTER }}$ & 3 & $5,465,115.7^{* *}$ & $22.4^{* *}$ & $13.9^{* *}$ & $0.76 \mathrm{~ns}$ & $545.2^{* *}$ & $53.8 \mathrm{~ns}$ & $0.04 *$ & $0.22 *$ & $0.6^{* *}$ & - \\
\hline SITE $\times$ SCA & 186 & $925,393.7 \mathrm{~ns}$ & $2.2 \mathrm{~ns}$ & $2.3 \mathrm{~ns}$ & $0.53 \mathrm{~ns}$ & $138.2 \mathrm{~ns}$ & $65.3 \mathrm{~ns}$ & $0.01 \mathrm{~ns}$ & $0.09 \mathrm{~ns}$ & $0.1 \mathrm{~ns}$ & - \\
\hline RESIDUALS & 442 & $839,707.3$ & 2 & 2.2 & 0.61 & 127.6 & 75 & 0.01 & 0.08 & 0.1 & - \\
\hline \%GCA SS & & 53.4 & 56.3 & 54.0 & 26.8 & 72.4 & 57.4 & 37.1 & 44.7 & 45.6 & - \\
\hline$\%$ SCA SS & & 46.6 & 43.7 & 46.0 & 73.2 & 27.6 & 42.6 & 62.9 & 55.3 & 54.4 & - \\
\hline
\end{tabular}

Days to silking; ASI = Anthesis silking interval; PLHT = Plant height; PASP = Plant aspect; EHT = Ear height; EASP = Ear aspect; EPP = Ears per plant and LD = leaf death. 
Table 2. Grain yield and other agronomic traits of hybrids (the best 15 and the worst 10 based on the base index) and hybrid checks evaluated under drought (DT) and optimal (OP) environments in Nigeria between 2013 and 2014

\begin{tabular}{|c|c|c|c|c|c|c|c|c|c|c|c|c|c|c|c|c|c|c|}
\hline \multirow[b]{2}{*}{ Pedigree } & \multicolumn{2}{|c|}{ GY } & \multicolumn{2}{|c|}{ ASI } & \multicolumn{2}{|c|}{ PLHT } & \multicolumn{2}{|c|}{ EHT } & \multicolumn{2}{|c|}{ PASP } & \multicolumn{2}{|c|}{ EASP } & \multicolumn{2}{|c|}{ EPP } & \multirow[b]{2}{*}{ LD } & \multirow[b]{2}{*}{$\mathbf{Y R}$} & \multirow[b]{2}{*}{ BI } & \multirow[b]{2}{*}{$\begin{array}{l}\text { Reaction to } \\
\text { Drought Using } \\
\text { Base Index }\end{array}$} \\
\hline & DT & OP & DT & OP & DT & OP & DT & OP & DT & OP & DT & OP & DT & OP & & & & \\
\hline TZdEEI $54 \times$ TZEEI 13 & 3508 & 7028 & 2 & 1 & 152 & 193 & 64 & 97 & 5 & 2 & 3 & 2 & 0.9 & 1.1 & 3 & 50.1 & 14.96 & Tolerant \\
\hline TZdEEI $91 \times$ TZEEI 21 & 4480 & 6210 & 2 & 1 & 151 & 207 & 67 & 101 & 5 & 3 & 3 & 3 & 0.9 & 1.0 & 3 & 27.9 & 14.48 & Tolerant \\
\hline TZdEEI $55 \times$ TZEEI 21 & 4078 & 6755 & 3 & 1 & 170 & 209 & 73 & 98 & 4 & 3 & 4 & 3 & 1.0 & 1.0 & 3 & 39.6 & 13.20 & Tolerant \\
\hline TZdEEI $23 \times$ TZEEI 14 & 3584 & 5537 & 2 & 1 & 145 & 181 & 72 & 92 & 5 & 3 & 4 & 3 & 1.0 & 0.9 & 3 & 35.3 & 12.59 & Tolerant \\
\hline TZdEEI $23 \times$ TZEEI 21 & 3195 & 6473 & 2 & 1 & 147 & 189 & 67 & 97 & 4 & 2 & 4 & 3 & 0.9 & 1.0 & 4 & 50.6 & 11.97 & Tolerant \\
\hline TZdEEI $70 \times$ TZEEI 14 & 2680 & 6603 & 2 & 1 & 139 & 181 & 73 & 95 & 5 & 3 & 3 & 3 & 1.0 & 1.1 & 4 & 59.4 & 11.95 & Tolerant \\
\hline TZdEEI $50 \times$ TZEEI 21 & 3257 & 5777 & 2 & 1 & 143 & 179 & 67 & 95 & 5 & 3 & 5 & 3 & 0.9 & 0.9 & 4 & 43.6 & 11.45 & Tolerant \\
\hline TZdEEI $51 \times$ TZEEI 13 & 3678 & 6035 & 2 & 2 & 148 & 180 & 78 & 105 & 5 & 3 & 4 & 3 & 0.9 & 1.1 & 3 & 39.1 & 11.31 & Tolerant \\
\hline TZdEEI $71 \times$ TZEEI 29 & 3156 & 7095 & 3 & 1 & 147 & 184 & 77 & 98 & 5 & 3 & 4 & 2 & 0.8 & 1.0 & 4 & 55.5 & 11.01 & Tolerant \\
\hline TZdEEI $21 \times$ TZEEI 21 & 2641 & 7128 & 2 & 1 & 133 & 164 & 60 & 87 & 5 & 3 & 5 & 3 & 0.9 & 1.0 & 4 & 62.9 & 10.96 & Tolerant \\
\hline TZdEEI $95 \times$ TZEEI 14 & 3497 & 5871 & 2 & 2 & 140 & 176 & 69 & 93 & 4 & 3 & 3 & 3 & 0.9 & 0.9 & 4 & 40.4 & 10.26 & Tolerant \\
\hline TZdEEI $111 \times$ TZEEI 14 & 3222 & 5968 & 2 & 1 & 145 & 187 & 73 & 94 & 5 & 3 & 4 & 3 & 0.9 & 1.1 & 4 & 46.0 & 10.26 & Tolerant \\
\hline TZdEEI $55 \times$ TZEEI 13 & 2497 & 8136 & 3 & 1 & 150 & 200 & 70 & 99 & 5 & 2 & 5 & 2 & 0.9 & 1.0 & 3 & 69.3 & 10.07 & Tolerant \\
\hline TZdEEI $64 \times$ TZEEI 21 & 2764 & 5877 & 2 & 1 & 155 & 193 & 77 & 100 & 5 & 3 & 4 & 3 & 0.9 & 1.1 & 4 & 53.0 & 9.71 & Tolerant \\
\hline TZdEEI $74 \times$ TZEEI 13 & 2973 & 5659 & 2 & 1 & 140 & 167 & 75 & 93 & 5 & 3 & 4 & 3 & 0.9 & 1.0 & 4 & 47.5 & 9.71 & Tolerant \\
\hline TZdEEI $83 \times$ TZEEI 13 & 1524 & 5111 & 3 & 2 & 129 & 169 & 63 & 96 & 7 & 3 & 6 & 3 & 0.7 & 0.9 & 4 & 70.2 & -5.13 & Susceptible \\
\hline TZdEEI $18 \times$ TZEEI 29 & 1957 & 5153 & 3 & 1 & 135 & 177 & 56 & 86 & 6 & 3 & 6 & 3 & 0.8 & 1.0 & 4 & 62.0 & -5.69 & Susceptible \\
\hline TZdEEI $81 \times$ TZEEI 29 & 2335 & 2410 & 3 & 2 & 131 & 166 & 65 & 89 & 6 & 4 & 5 & 4 & 0.9 & 0.9 & 4 & 3.1 & -5.69 & Susceptible \\
\hline TZdEEI $83 \times$ TZEEI 21 & 1708 & 4586 & 3 & 1 & 141 & 179 & 62 & 86 & 6 & 3 & 6 & 3 & 0.8 & 0.8 & 4 & 62.8 & -5.71 & Susceptible \\
\hline TZdEEI $97 \times$ TZEEI 13 & 1928 & 3137 & 2 & 1 & 144 & 167 & 65 & 82 & 5 & 3 & 6 & 3 & 0.8 & 0.7 & 4 & 38.6 & -5.75 & Susceptible \\
\hline TZdEEI $94 \times$ TZEEI 29 & 2011 & 5067 & 4 & 1 & 141 & 188 & 67 & 95 & 6 & 3 & 6 & 3 & 0.7 & 1.0 & 4 & 60.3 & -6.82 & Susceptible \\
\hline TZdEEI $90 \times$ TZEEI 13 & 1229 & 4781 & 3 & 2 & 143 & 180 & 65 & 87 & 6 & 3 & 6 & 3 & 0.6 & 0.9 & 4 & 74.3 & -8.24 & Susceptible \\
\hline TZdEEI $42 \times$ TZEEI 13 & 1956 & 3142 & 4 & 1 & 130 & 161 & 66 & 83 & 6 & 3 & 6 & 3 & 0.8 & 0.9 & 4 & 37.8 & -8.44 & Susceptible \\
\hline TZdEEI $107 \times$ TZEEI 21 & 1592 & 2219 & 2 & 1 & 137 & 176 & 70 & 88 & 7 & 3 & 6 & 3 & 0.9 & 0.7 & 4 & 28.3 & -9.15 & Susceptible \\
\hline TZdEEI $83 \times$ TZEEI 29 & 1340 & 4980 & 5 & 1 & 135 & 166 & 63 & 74 & 7 & 3 & 6 & 3 & 0.6 & 1.0 & 4 & 73.1 & -10.3 & Susceptible \\
\hline CHECK 1 -TZEEI $21 \times$ TZEEI 29 & 2672 & 5851 & 3 & 1 & 145 & 192 & 69 & 97 & 6 & 3 & 5 & 3 & 0.9 & 0.9 & 4 & 54.3 & 6.35 & Susceptible \\
\hline CHECK 2-TZEEI $32 \times$ TZEEI 13 & 1750 & 4083 & 3 & 1 & 140 & 172 & 64 & 80 & 6 & 3 & 6 & 3 & 0.7 & 1.0 & 4 & 57.1 & -6.81 & Susceptible \\
\hline CHECK 3-(TZEEI $21 \times$ TZEEI 14) $\times$ TZEEI 29 & 2745 & 5528 & 3 & 1 & 145 & 183 & 74 & 93 & 5 & 3 & 5 & 3 & 0.9 & 0.9 & 3 & 50.3 & 1.7 & Tolerant \\
\hline $\begin{array}{c}\text { CHECK } 4-(\text { TZEEI } 29 \times \text { TZEEI } 21) \times(\text { TZEEI } \\
14 \times \text { TZEEI 37) }\end{array}$ & 3004 & 4190 & 2 & 1 & 142 & 167 & 64 & 80 & 5 & 3 & 5 & 3 & 0.9 & 0.9 & 4 & 28.3 & 1.88 & Tolerant \\
\hline
\end{tabular}

$\mathrm{GY}=$ grain yield $\left(\mathrm{t} \mathrm{ha}^{-1}\right) ; \mathrm{DA}=$ Days to anthesis; $\mathrm{DS}=$ Days to silking; $\mathrm{ASI}=$ Anthesis-silking interval; PLHT = Plant height $(\mathrm{cm}) ;$

EASP $=$ Ear aspect (Scale 1-9); EPP = Ears per plant and LD = leaf death (Scale 1-9); YR = yield reduction and BI = base index 
Table 3. Grain yield and yield reduction or increase based on the best check under drought and optimal conditions (best 15 and the worst 10 ).

\begin{tabular}{|c|c|c|c|c|}
\hline \multirow{2}{*}{ Pedigree } & \multicolumn{2}{|c|}{ Grain Yield (kg ha $\left.{ }^{-1}\right)$} & \multicolumn{2}{|c|}{ Yield Difference (\%) Based on the Best Check } \\
\hline & Drought & Optimal & Drought & Optimal \\
\hline \multicolumn{5}{|l|}{ Best 15 hybrids } \\
\hline TZdEEI $54 \times$ TZEEI 13 & 3508 & 7028 & 16.78 & 20.12 \\
\hline TZdEEI $91 \times$ TZEEI 21 & 4480 & 6210 & 49.13 & 6.14 \\
\hline TZdEEI $55 \times$ TZEEI 21 & 4078 & 6755 & 35.75 & 15.45 \\
\hline TZdEEI $23 \times$ TZEEI 14 & 3584 & 5537 & 19.31 & -5.37 \\
\hline TZdEEI $23 \times$ TZEEI 21 & 3195 & 6473 & 6.36 & 10.63 \\
\hline TZdEEI $70 \times$ TZEEI 14 & 2680 & 6603 & -10.79 & 12.85 \\
\hline TZdEEI $50 \times$ TZEEI 21 & 3257 & 5777 & 8.42 & -1.26 \\
\hline TZdEEI $51 \times$ TZEEI 13 & 3678 & 6035 & 22.44 & 3.14 \\
\hline TZdEEI $71 \times$ TZEEI 29 & 3156 & 7095 & 5.06 & 21.26 \\
\hline TZdEEI $21 \times$ TZEEI 21 & 2641 & 7128 & -12.08 & 21.83 \\
\hline TZdEEI $95 \times$ TZEEI 14 & 3497 & 5871 & 16.41 & 0.34 \\
\hline TZdEEI $111 \times$ TZEEI 14 & 3222 & 5968 & 7.26 & 2.00 \\
\hline TZdEEI $55 \times$ TZEEI 13 & 2497 & 8136 & -16.88 & 39.05 \\
\hline TZdEEI $64 \times$ TZEEI 21 & 2764 & 5877 & -7.99 & 0.44 \\
\hline TZdEEI $74 \times$ TZEEI 13 & 2973 & 5659 & -1.03 & -3.28 \\
\hline \multicolumn{5}{|l|}{ Worst 10 hybrids } \\
\hline TZdEEI $83 \times$ TZEEI 13 & 1524 & 5111 & -49.27 & -12.65 \\
\hline TZdEEI $18 \times$ TZEEI 29 & 1957 & 5153 & -34.85 & -11.93 \\
\hline TZdEEI $81 \times$ TZEEI 29 & 2335 & 2410 & -22.27 & -58.81 \\
\hline TZdEEI $83 \times$ TZEEI 21 & 1708 & 4586 & -43.14 & -21.62 \\
\hline TZdEEI $97 \times$ TZEEI 13 & 1928 & 3137 & -35.82 & -46.39 \\
\hline TZdEEI $94 \times$ TZEEI 29 & 2011 & 5067 & -33.06 & -13.40 \\
\hline TZdEEI $90 \times$ TZEEI 13 & 1229 & 4781 & -59.09 & -18.29 \\
\hline TZdEEI $42 \times$ TZEEI 13 & 1956 & 3142 & -34.89 & -46.30 \\
\hline TZdEEI $107 \times$ TZEEI 21 & 1592 & 2219 & -47.00 & -62.07 \\
\hline TZdEEI $83 \times$ TZEEI 29 & 1340 & 4980 & -55.39 & -14.89 \\
\hline \multicolumn{5}{|l|}{ Hybrid checks } \\
\hline CHECK 1-TZEEI $21 \times$ TZEEI 29 & 2672 & 5851 & -11.05 & 0.00 \\
\hline CHECK 2 -TZEEI $32 \times$ TZEEI 13 & 1750 & 4083 & -41.74 & -30.22 \\
\hline CHECK 3-(TZEEI $21 \times$ TZEEI 14$) \times$ TZEEI 29 & 2745 & 5528 & -8.62 & -5.52 \\
\hline CHECK 4-(TZEEI $29 \times$ TZEEI 21) $\times($ TZEEI $14 \times$ TZEEI 37) & 3004 & 4190 & 0.00 & -28.39 \\
\hline
\end{tabular}




\subsection{Variance Components and Heritability of Traits under Drought and Optimal Conditions}

The genotypic variance and its components, estimates of heritability for grain yield and other measured traits of the extra-early maturing maize hybrids showed that the additive genetic variance estimates were high for DA, PLHT, EHT and grain yield under managed drought and optimal conditions (Table 4). Heritability for ASI was generally low under optimal conditions. EPP also recorded low broad sense and narrow sense heritability estimates under drought and optimal conditions (Table 4). Narrow sense heritability was higher for grain yield under drought compared with optimal conditions.

Table 4. Estimates of variance components, heritability and genetic gains under drought (2013 and 2014 seasons at Ikenne) and optimal (2014 cropping season at Ikenne and Bagauda).

\begin{tabular}{|c|c|c|c|c|c|c|c|c|c|c|}
\hline \multirow[t]{2}{*}{ Trait $^{+}$} & \multicolumn{2}{|c|}{ Line Variance } & \multicolumn{2}{|c|}{ Tester Variance } & \multicolumn{2}{|c|}{ Line $\times$ Tester Variance } & \multicolumn{2}{|c|}{$\begin{array}{c}\text { Genotype Variance } \\
\left(\sigma_{\mathrm{G}}{ }^{2}\right)\end{array}$} & \multicolumn{2}{|c|}{ Additive Variance } \\
\hline & Drought & Optimal & Drought & Optimal & Drought & Optimal & Drought & Optimal & Drought & Optimal \\
\hline ASI & 0.11 & 0.00 & 0.01 & 0.00 & 0.07 & 0.00 & 0.07 & 0.00 & 0.28 & 0.00 \\
\hline EASP & 0.10 & 0.02 & 0.04 & 0.01 & 0.06 & 0.01 & 0.07 & 0.01 & 0.29 & 0.05 \\
\hline EHT & 6.17 & 15.07 & 2.75 & 2.74 & 0.40 & 4.17 & 4.69 & 9.73 & 18.76 & 38.94 \\
\hline EPP & 0.00 & 0.00 & 0.00 & 0.00 & 0.00 & 0.00 & 0.00 & 0.00 & 0.00 & 0.00 \\
\hline DA & 0.55 & 0.43 & 0.00 & 0.17 & 0.23 & 0.29 & 0.31 & 0.32 & 1.23 & 1.27 \\
\hline GY & $93,643.32$ & $244,727.78$ & $19,920.99$ & 660.92 & $38,771.41$ & $200,496.05$ & $61,758.83$ & $139,170.26$ & $247,035.32$ & $556,681.05$ \\
\hline \multirow[t]{3}{*}{ LD } & 0.01 & - & 0.05 & - & 0.03 & - & 0.03 & - & 0.12 & - \\
\hline & \multicolumn{2}{|c|}{ Dominance Variance } & \multicolumn{2}{|c|}{$\begin{array}{l}\text { Environmental } \\
\text { Variance }\left(\sigma_{\mathrm{e}}^{2}\right)\end{array}$} & \multicolumn{2}{|c|}{$\begin{array}{c}\text { Broad Sense } \\
\text { Heritability }\left(\mathbf{H}^{2}\right)\end{array}$} & \multicolumn{2}{|c|}{$\begin{array}{c}\text { Narrow Sense } \\
\text { Heritability }\left(h^{2}\right)\end{array}$} & & \\
\hline & Drought & Optimal & Drought & Optimal & Drought & Optimal & Drought & Optimal & & \\
\hline EHT & 1.60 & 16.69 & 70.61 & 76.23 & 0.22 & 0.42 & 0.21 & 0.30 & & \\
\hline EPP & 0.00 & 0.00 & 0.02 & 0.01 & 0.31 & 0.31 & 0.09 & 0.12 & & \\
\hline PASP & 0.00 & 0.05 & 0.65 & 0.10 & 0.34 & 0.42 & 0.34 & 0.15 & & \\
\hline PLHT & 0.00 & 34.10 & 140.34 & 144.48 & 0.38 & 0.57 & 0.38 & 0.47 & & \\
\hline DA & 0.92 & 1.18 & 2.48 & 2.40 & 0.46 & 0.50 & 0.27 & 0.26 & & \\
\hline GY & $155,085.66$ & $801,984.18$ & $482,776.39$ & $967,317.88$ & 0.45 & 0.58 & 0.28 & 0.24 & & \\
\hline LD & 0.12 & - & 0.49 & - & 0.33 & - & 0.16 & - & & \\
\hline
\end{tabular}

\subsection{Estimates of GCA and SCA Effects}

The inbred lines TZdEEI 51 and TZdEEI 91 had positive and significant $(p \leq 0.05)$ GCA effects for grain yield under drought. Similarly, the inbred lines TZdEEI 55 displayed positive and significant $(p \leq 0.05)$ GCA effects for grain yield under optimal growing conditions (Table 5). Additionally, significant GCA effects were observed for some yield-related traits, which are components of the IITA drought-tolerance base index. For example, significant and negative GCA effects were recorded for PASP for TZdEEI 23 under both drought and optimal conditions, for PASP and EASP for TZdEEI 71 under drought, and positive and significant GCA effects for EPP for TZdEEI 70 under both drought and optimal conditions. 
Table 5. General combining ability (GCA) effects of lines and testers for grain yield and other agronomic traits under drought (DT) and optimal (OPT) conditions.

\begin{tabular}{|c|c|c|c|c|c|c|c|c|c|c|}
\hline \multirow{2}{*}{ Lines } & \multicolumn{2}{|c|}{$\mathrm{ASI}^{\dagger}$} & \multicolumn{2}{|c|}{ Days to Silking } & \multicolumn{2}{|c|}{ Ear Aspect } & \multicolumn{2}{|c|}{ Ear Height } & \multicolumn{2}{|c|}{ Ears Per Plant } \\
\hline & DT & OPT & DT & OPT & DT & OPT & DT & OPT & DT & OPT \\
\hline TZdEEI 16 & -0.11 & -0.22 & -0.15 & -0.81 & 0.05 & -0.17 & 0.89 & 8.78 & -0.04 & 0.07 \\
\hline TZdEEI 17 & -0.25 & 0.09 & -1.62 & -0.68 & 0.08 & -0.05 & 2.95 & 3.42 & 0.00 & -0.03 \\
\hline TZdEEI 18 & -0.10 & -0.10 & -0.49 & 0.06 & 0.53 & -0.05 & -4.45 & -1.84 & -0.04 & 0.02 \\
\hline TZdEEI 20 & -0.10 & 0.21 & $-2.26^{*}$ & $-1.89 * *$ & 0.31 & -0.07 & -5.58 & -8.36 & 0.01 & -0.03 \\
\hline TZdEEI 21 & -0.34 & -0.22 & -0.19 & -0.92 & -0.30 & -0.18 & -2.19 & -1.04 & 0.03 & 0.05 \\
\hline TZdEEI 22 & -0.68 & -0.16 & -1.10 & -0.33 & -0.17 & 0.10 & 2.42 & -0.94 & 0.02 & 0.02 \\
\hline TZdEEI 23 & -0.21 & -0.22 & -0.50 & 0.35 & -0.47 & -0.21 & 1.56 & 3.97 & 0.05 & -0.03 \\
\hline TZdEEI 24 & -0.34 & -0.10 & -1.38 & -0.70 & 0.31 & 0.27 & -1.74 & -7.66 & 0.00 & -0.06 \\
\hline TZdEEI 25 & -0.15 & -0.22 & -1.21 & -0.64 & -0.13 & 0.14 & 0.26 & -3.69 & 0.01 & -0.05 \\
\hline TZdEEI 26 & 0.35 & 0.03 & -0.30 & -0.92 & 0.29 & 0.05 & 1.11 & 3.32 & 0.00 & 0.01 \\
\hline TZdEEI 31 & 0.10 & 0.21 & 0.16 & -0.47 & $0.97 * *$ & 0.10 & -1.42 & 0.30 & -0.10 & 0.01 \\
\hline TZdEEI 33 & -0.09 & 0.28 & 1.96 & 0.98 & 0.40 & -0.26 & 2.78 & $15.63 * *$ & -0.09 & 0.01 \\
\hline TZdEEI 34 & -0.05 & 0.21 & 1.17 & 0.35 & -0.05 & 0.01 & -0.05 & 3.75 & 0.04 & 0.05 \\
\hline TZdEEI 42 & 0.23 & -0.22 & -0.32 & 0.40 & 0.38 & 0.28 & -0.21 & -4.07 & -0.02 & -0.01 \\
\hline TZdEEI 43 & 0.33 & -0.10 & 0.24 & 0.45 & -0.31 & $0.35^{*}$ & -1.20 & -6.41 & 0.00 & 0.00 \\
\hline TZdEEI 44 & 0.19 & -0.10 & -0.61 & -1.36 & 0.55 & 0.11 & -0.63 & -3.43 & -0.05 & -0.04 \\
\hline TZdEEI 45 & -0.10 & -0.29 & -1.08 & -1.05 & -0.25 & 0.19 & 0.50 & 0.65 & 0.07 & 0.03 \\
\hline TZdEEI 46 & -0.20 & -0.29 & -1.12 & 1.15 & -0.20 & 0.07 & 1.13 & -1.36 & 0.03 & -0.02 \\
\hline TZdEEI 47 & -0.42 & 0.03 & -0.75 & -0.54 & -0.27 & -0.03 & -1.47 & -6.63 & 0.03 & 0.02 \\
\hline TZdEEI 50 & -0.16 & -0.10 & -0.35 & -0.03 & -0.57 & 0.02 & 2.47 & -0.20 & 0.03 & -0.03 \\
\hline TZdEEI 51 & -0.47 & -0.04 & -0.24 & 0.88 & -0.66 & -0.07 & 3.70 & 2.31 & 0.05 & 0.01 \\
\hline TZdEEI 54 & 0.09 & 0.21 & -0.02 & -1.30 & -0.44 & -0.27 & -1.26 & 2.16 & 0.00 & 0.00 \\
\hline TZdEEI 55 & 0.69 & -0.16 & 0.18 & -1.36 & -0.51 & -0.26 & 1.10 & 1.73 & 0.07 & 0.00 \\
\hline TZdEEI 56 & -0.57 & 0.34 & -0.46 & 1.04 & -0.31 & 0.00 & 2.57 & -1.19 & 0.01 & 0.04 \\
\hline TZdEEI 58 & -0.10 & 0.21 & -0.57 & -0.12 & -0.30 & -0.16 & -0.97 & 1.93 & -0.03 & 0.03 \\
\hline TZdEEI 59 & 0.17 & 0.09 & -0.34 & 0.84 & 0.06 & 0.10 & -1.02 & 0.94 & 0.01 & 0.06 \\
\hline TZdEEI 61 & 0.39 & -0.16 & -1.01 & -0.95 & 0.18 & 0.08 & $-9.89 * *$ & $-11.68^{* *}$ & -0.03 & 0.00 \\
\hline TZdEEI 62 & -0.23 & 0.15 & -0.69 & 0.54 & -0.46 & -0.07 & 0.35 & 0.58 & 0.02 & 0.01 \\
\hline TZdEEI 64 & -0.54 & 0.03 & -0.91 & -0.20 & -0.50 & 0.05 & $7.54 *$ & 3.66 & 0.03 & 0.08 \\
\hline TZdEEI 66 & -0.35 & 0.09 & -0.43 & 0.42 & 0.01 & 0.27 & 0.53 & -7.89 & -0.02 & -0.04 \\
\hline TZdEEI 68 & 0.27 & 0.09 & 1.45 & 0.75 & 0.00 & -0.12 & -4.70 & -0.51 & -0.03 & -0.01 \\
\hline TZdEEI 69 & 0.00 & -0.10 & 0.54 & 1.16 & -0.43 & -0.19 & 2.99 & 5.24 & -0.03 & -0.04 \\
\hline TZdEEI 70 & -0.78 & -0.29 & -0.57 & -0.35 & -0.53 & -0.27 & -0.79 & -1.66 & $0.09 *$ & $0.09 *$ \\
\hline TZdEEI 71 & -0.15 & 0.03 & 0.62 & 0.58 & $-0.76^{*}$ & -0.34 * & 4.69 & $10.56^{*}$ & 0.01 & 0.01 \\
\hline TZdEEI 72 & 0.52 & 0.03 & $2.39 *$ & 1.14 & -0.59 & -0.14 & -2.98 & 0.62 & 0.03 & 0.08 \\
\hline TZdEEI 73 & 0.36 & -0.10 & 0.15 & 0.26 & 0.34 & 0.28 & 2.79 & 5.04 & 0.02 & 0.06 \\
\hline TZdEEI 74 & -0.80 & -0.04 & -1.80 & -0.17 & -0.17 & -0.03 & 5.49 & -3.03 & 0.05 & 0.01 \\
\hline TZdEEI 75 & -0.40 & -0.04 & -0.23 & 0.71 & -0.29 & -0.12 & 3.35 & 4.76 & 0.06 & 0.05 \\
\hline TZdEEI 76 & -0.22 & -0.22 & 0.62 & -0.15 & -0.28 & -0.34 * & -1.92 & 3.13 & -0.04 & $0.09 *$ \\
\hline TZdEEI 78 & -0.14 & 0.09 & -0.35 & -0.78 & 0.19 & 0.15 & 1.60 & -3.19 & -0.02 & -0.05 \\
\hline TZdEEI 80 & -0.21 & -0.29 & 0.59 & 0.43 & 0.04 & 0.14 & -2.04 & -0.80 & -0.06 & 0.00 \\
\hline TZdEEI 81 & -0.11 & 0.09 & -0.76 & 0.86 & 0.16 & 0.08 & 3.94 & 2.65 & 0.03 & -0.01 \\
\hline TZdEEI 82 & 0.08 & -0.04 & 0.33 & 0.00 & 0.29 & 0.17 & 1.82 & -3.75 & -0.01 & -0.02 \\
\hline TZdEEI 83 & $1.21^{* *}$ & -0.10 & $2.88 *$ & 0.66 & $0.89 *$ & 0.06 & -4.13 & -3.90 & $-0.12^{* *}$ & -0.06 \\
\hline TZdEEI 84 & 0.35 & -0.29 & 0.17 & 0.84 & 0.26 & 0.12 & -0.43 & -3.23 & 0.02 & -0.09 * \\
\hline TZdEEI 85 & 0.54 & -0.04 & 1.27 & 0.25 & 0.50 & -0.08 & -4.10 & -4.90 & -0.07 & -0.05 \\
\hline TZdEEI 89 & -0.17 & -0.16 & -1.23 & -1.36 & -0.09 & -0.06 & 1.56 & -3.81 & 0.05 & 0.02 \\
\hline TZdEEI 90 & 0.82 & 0.28 & 0.92 & -0.32 & 0.19 & -0.10 & -1.23 & -5.94 & 0.03 & -0.01 \\
\hline TZdEEI 91 & -0.48 & 0.09 & -0.48 & 0.09 & -0.64 & -0.21 & 4.52 & 6.58 & 0.07 & 0.04 \\
\hline TZdEEI 94 & $0.85 *$ & -0.16 & 1.06 & -0.56 & 0.21 & -0.09 & -1.96 & 2.92 & -0.07 & 0.02 \\
\hline TZdEEI 95 & 0.53 & 0.21 & -0.91 & 0.40 & -0.23 & 0.13 & -3.19 & -0.06 & 0.04 & -0.07 \\
\hline TZdEEI 96 & 0.21 & 0.15 & -0.23 & -0.04 & 0.02 & 0.20 & 0.18 & 0.54 & 0.02 & -0.03 \\
\hline TZdEEI 97 & -0.53 & -0.04 & -0.10 & 0.26 & -0.14 & 0.04 & -2.11 & -0.29 & 0.02 & -0.05 \\
\hline TZdEEI 99 & 0.52 & 0.46 & 1.68 & 2.06 & 0.27 & 0.14 & -5.19 & -1.60 & -0.03 & 0.01 \\
\hline TZdEEI 100 & -0.44 & 0.21 & -0.43 & 0.46 & 0.50 & 0.11 & -4.66 & -0.83 & 0.02 & 0.00 \\
\hline TZdEEI 102 & -0.44 & 0.15 & -0.42 & -0.85 & 0.62 & 0.19 & -3.68 & -3.07 & -0.06 & -0.02 \\
\hline TZdEEI 103 & -0.11 & 0.03 & -0.72 & -0.92 & 0.03 & 0.20 & -1.05 & 1.65 & 0.03 & 0.05 \\
\hline TZdEEI 104 & 0.04 & 0.28 & 1.14 & 0.39 & 0.16 & -0.03 & -2.65 & 2.48 & 0.00 & -0.04 \\
\hline TZdEEI 105 & 0.15 & -0.16 & 2.02 & -0.22 & 0.36 & -0.05 & -5.11 & -2.69 & -0.03 & -0.06 \\
\hline TZdEEI 106 & 0.60 & -0.16 & 0.82 & -0.27 & 0.38 & -0.22 & 5.67 & 3.22 & -0.02 & -0.03 \\
\hline TZdEEI 107 & $1.21^{* *}$ & -0.10 & $3.20 * *$ & 0.73 & 0.56 & -0.01 & 4.92 & 0.90 & -0.04 & -0.02 \\
\hline TZdEEI 108 & 0.08 & 0.34 & 1.41 & 0.63 & 0.29 & 0.07 & 3.37 & 3.72 & -0.04 & -0.09 * \\
\hline TZdEEI 111 & -0.29 & 0.34 & -0.59 & 0.03 & -0.37 & -0.03 & 5.49 & 4.28 & 0.00 & 0.02 \\
\hline TZEEI 13 & 0.05 & -0.04 & 0.09 & 0.13 & 0.13 & 0.06 & -0.37 & 0.72 & -0.01 & 0.03 \\
\hline TZEEI 14 & -0.14 & 0.08 & -0.20 & 0.47 & -0.29 & -0.03 & 2.37 & 1.16 & 0.03 & 0.01 \\
\hline TZEEI 21 & -0.09 & -0.06 & -0.20 & -0.49 & 0.03 & 0.08 & -1.97 & 0.85 & 0.00 & -0.01 \\
\hline TZEEI 29 & 0.18 & 0.03 & 0.31 & -0.12 & 0.13 & -0.12 & -0.02 & -2.61 & -0.01 & -0.02 \\
\hline SE Line & 0.43 & 0.19 & 1.06 & 0.76 & 0.39 & 0.16 & 3.20 & 4.52 & 0.04 & 0.04 \\
\hline SE Tester & 0.12 & 0.05 & 0.21 & 0.35 & 0.17 & 0.08 & 1.50 & 1.53 & 0.02 & 0.02 \\
\hline
\end{tabular}


Table 5. Cont.

\begin{tabular}{|c|c|c|c|c|c|c|c|c|c|}
\hline \multirow{2}{*}{ Lines } & \multicolumn{2}{|c|}{ Plant Aspect } & \multicolumn{2}{|c|}{ Plant Height } & \multicolumn{2}{|c|}{ Days to Anthesis } & \multicolumn{2}{|c|}{ Grain Yield } & \multirow[t]{2}{*}{ Leaf Death } \\
\hline & DT & OPT & DT & OPT & DT & OPT & DT & OPT & \\
\hline TZdEEI 16 & 0.01 & -0.20 & 6.86 & $15.63 *$ & 0.12 & -1.05 & -63.30 & 662.40 & 0.00 \\
\hline TZdEEI 17 & -0.42 & -0.11 & 4.27 & 0.03 & -1.48 & -0.65 & 70.28 & 234.50 & -0.08 \\
\hline TZdEEI 18 & 0.18 & -0.07 & 0.36 & 1.00 & -0.38 & -0.15 & -192.98 & 45.28 & -0.16 \\
\hline TZdEEI 20 & 0.04 & 0.01 & -0.28 & -2.61 & $-2.18 * *$ & $-1.85 *$ & -89.93 & 71.25 & $0.52 *$ \\
\hline TZdEEI 21 & -0.18 & -0.17 & -4.77 & -5.49 & 0.11 & -1.03 & 181.18 & 845.00 & -0.22 \\
\hline TZdEEI 22 & -0.02 & -0.02 & -7.82 & -0.91 & -0.34 & -0.39 & 313.35 & -288.00 & $-0.45^{*}$ \\
\hline TZdEEI 23 & $-0.85 *$ & -0.30 * & 9.85 & 10.50 & -0.37 & 0.34 & 575.58 & 717.40 & -0.42 \\
\hline TZdEEI 24 & 0.67 & 0.20 & -8.24 & $-16.59 *$ & -1.06 & -0.72 & -228.71 & -924.80 & 0.31 \\
\hline TZdEEI 25 & 0.30 & 0.15 & -5.03 & -130.00 & -1.10 & -0.76 & -22.08 & -309.20 & 0.04 \\
\hline TZdEEI 26 & 0.17 & 0.02 & -9.15 & -0.01 & -0.69 & -0.76 & -26.74 & -154.80 & -0.22 \\
\hline TZdEEI 31 & 0.55 & 0.08 & -1.88 & -3.43 & -0.02 & -0.12 & $-883.44^{* *}$ & -105.20 & 0.30 \\
\hline TZdEEI 33 & 0.44 & -0.17 & 0.59 & 16.95 * & $2.03^{* *}$ & 0.95 & -410.97 & 761.60 & -0.38 \\
\hline TZdEEI 34 & 0.02 & -0.14 & 2.97 & 6.03 & 1.22 & 0.82 & 303.43 & 548.70 & -0.11 \\
\hline TZdEEI 42 & 0.23 & 0.20 & -1.31 & -12.40 & -0.60 & 0.43 & -230.38 & $-1186.72 * *$ & 0.31 \\
\hline TZdEEI 43 & 0.13 & 0.20 & -3.83 & -9.65 & -0.09 & 0.55 & -147.29 & -993.30 & -0.28 \\
\hline TZdEEI 44 & 0.31 & 0.08 & -7.28 & -11.40 & -0.76 & $-1.61 *$ & -254.31 & -234.80 & 0.19 \\
\hline TZdEEI 45 & -0.38 & 0.01 & -7.31 & -2.24 & -0.98 & -1.25 & 530.79 & 355.80 & 0.09 \\
\hline TZdEEI 46 & -0.34 & 0.08 & -5.86 & -10.30 & -0.98 & 1.05 & 248.76 & 176.20 & -0.17 \\
\hline TZdEEI 47 & -0.25 & -0.01 & 2.43 & -3.98 & -0.31 & -0.44 & 606.02 & 337.60 & -0.11 \\
\hline TZdEEI 50 & -0.29 & 0.01 & 6.44 & -4.21 & -0.20 & -0.17 & 615.67 & 164.50 & 0.03 \\
\hline TZdEEI 51 & -0.58 & -0.02 & 3.49 & -5.42 & 0.60 & 0.92 & $773.69 *$ & 323.30 & -0.28 \\
\hline TZdEEI 54 & -0.53 & -0.26 * & 13.21 * & 15.84 * & -0.16 & -1.13 & 164.23 & 842.80 & -0.03 \\
\hline TZdEEI 55 & $-0.76^{*}$ & -0.20 & 15.79 * & 16.90 * & -0.45 & -1.34 & 561.39 & 1234.21 * & -0.31 \\
\hline TZdEEI 56 & -0.29 & 0.02 & 5.22 & 3.77 & 0.04 & 1.49 & 353.54 & -82.13 & -0.29 \\
\hline TZdEEI 58 & -0.15 & -0.04 & 1.52 & 1.61 & -0.50 & -0.05 & 41.56 & -86.22 & -0.11 \\
\hline TZdEEI 59 & -0.30 & -0.11 & -5.72 & -5.49 & -0.55 & 0.86 & -51.06 & 310.60 & 0.17 \\
\hline TZdEEI 61 & 0.41 & -0.07 & $-15.92 * *$ & $-16.51 *$ & -1.42 & -0.85 & -119.73 & 463.40 & -0.01 \\
\hline TZdEEI 62 & -0.05 & -0.08 & 0.29 & 1.95 & -0.46 & 0.79 & 340.67 & -233.10 & 0.09 \\
\hline TZdEEI 64 & -0.32 & -0.17 & 14.21 * & 12.40 & -0.38 & -0.28 & 396.67 & -45.65 & -0.15 \\
\hline TZdEEI 66 & -0.21 & 0.21 & 12.60 & 0.37 & -0.09 & 0.38 & 65.67 & -997.60 & -0.13 \\
\hline TZdEEI 68 & 0.21 & -0.20 & 0.13 & 2.86 & 1.18 & 0.58 & -178.25 & 522.20 & -0.17 \\
\hline TZdEEI 69 & -0.40 & -0.14 & 6.57 & 9.56 & 0.57 & 0.91 & 333.79 & 480.90 & -0.09 \\
\hline TZdEEI 70 & -0.14 & -0.14 & -7.95 & 0.97 & 0.19 & -0.72 & 66.65 & 710.30 & 0.18 \\
\hline TZdEEI 71 & -0.74 * & -0.04 & 7.23 & 10.40 & 0.73 & 0.72 & 329.69 & 1055.00 & -0.08 \\
\hline TZdEEI 72 & 0.21 & -0.01 & -0.07 & -6.04 & $1.89 *$ & 1.09 & 267.55 & 352.20 & -0.37 \\
\hline TZdEEI 73 & -0.01 & 0.30 & 3.31 & 7.29 & -0.25 & 0.25 & -196.45 & $-1135.57^{*}$ & 0.05 \\
\hline TZdEEI 74 & -0.43 & -0.14 & 0.92 & -11.50 & -1.00 & -0.33 & 333.19 & 99.71 & 0.10 \\
\hline TZdEEI 75 & -0.20 & -0.14 & 1.53 & 2.23 & 0.10 & 0.77 & 257.09 & 40.40 & -0.19 \\
\hline TZdEEI 76 & 0.11 & -0.11 & -7.04 & 6.01 & 0.81 & -0.12 & -99.30 & 609.40 & -0.18 \\
\hline TZdEEI 78 & 0.63 & 0.17 & -6.90 & -6.21 & -0.31 & -0.41 & -214.19 & -955.70 & 0.08 \\
\hline TZdEEI 80 & 0.33 & 0.05 & -5.77 & -9.50 & 0.71 & 0.27 & -149.37 & -477.30 & -0.05 \\
\hline TZdEEI 81 & -0.20 & 0.11 & -0.81 & -1.12 & -0.62 & 1.03 & -180.26 & -734.30 & 0.32 \\
\hline TZdEEI 82 & 0.10 & 0.11 & 2.27 & -3.51 & 0.26 & -0.08 & -234.85 & -1015.00 & 0.26 \\
\hline TZdEEI 83 & $0.80 *$ & 0.02 & -3.71 & -5.70 & $1.67^{*}$ & 0.48 & $-870.26^{* *}$ & -433.00 & 0.39 \\
\hline TZdEEI 84 & -0.02 & 0.18 & 2.74 & -9.29 & -0.19 & 0.61 & -218.48 & -704.00 & 0.28 \\
\hline TZdEEI 85 & 0.37 & -0.01 & -9.14 & -9.48 & 0.64 & 0.24 & -551.55 & 472.60 & $0.69^{* *}$ \\
\hline TZdEEI 89 & -0.36 & -0.08 & 6.16 & 4.78 & -1.04 & -1.40 & 126.83 & 242.10 & 0.20 \\
\hline TZdEEI 90 & 0.31 & 0.05 & 9.54 & 8.12 & 0.19 & 0.09 & -209.21 & 279.30 & -0.02 \\
\hline TZdEEI 91 & -0.45 & -0.17 & 9.23 & 13.70 & 0.13 & -0.33 & $762.49 *$ & 942.70 & -0.24 \\
\hline TZdEEI 94 & 0.19 & -0.01 & -0.66 & 3.34 & 0.17 & -0.44 & -257.13 & 309.50 & 0.03 \\
\hline TZdEEI 95 & -0.41 & -0.05 & -9.05 & -6.45 & -1.45 & 0.53 & -41.81 & -29.43 & -0.02 \\
\hline TZdEEI 96 & -0.34 & 0.24 & 8.89 & 4.18 & -0.46 & 0.32 & 46.06 & -618.70 & 0.00 \\
\hline TZdEEI 97 & -0.22 & 0.14 & 4.02 & 4.96 & 0.36 & 0.50 & -24.32 & -616.60 & 0.15 \\
\hline TZdEEI 99 & 0.46 & 0.24 & -5.50 & 4.01 & 1.20 & $2.06^{* *}$ & -318.27 & -853.60 & -0.03 \\
\hline TZdEEI 100 & 0.24 & 0.08 & -3.78 & 3.57 & 0.08 & 0.53 & -356.58 & -31.39 & 0.17 \\
\hline TZdEEI 102 & 0.32 & 0.11 & -11.50 & -5.49 & 0.10 & -0.92 & -291.24 & -505.50 & 0.15 \\
\hline TZdEEI 103 & -0.08 & -0.02 & -7.07 & -2.00 & -0.62 & -0.98 & 102.31 & -60.26 & -0.02 \\
\hline TZdEEI 104 & 0.16 & -0.02 & -1.28 & 5.61 & 1.06 & 0.51 & -281.83 & 115.80 & -0.01 \\
\hline TZdEEI 105 & 0.56 & 0.05 & -4.74 & -6.10 & $1.88^{*}$ & -0.22 & -528.53 & -233.70 & 0.10 \\
\hline TZdEEI 106 & 0.10 & -0.10 & 6.32 & 8.73 & 0.15 & -0.46 & -272.99 & 705.10 & -0.07 \\
\hline TZdEEI 107 & $0.86^{*}$ & 0.17 & -0.68 & -2.18 & $2.01 *$ & 0.26 & -656.13 & -728.20 & 0.26 \\
\hline TZdEEI 108 & 0.60 & 0.20 & -3.03 & -5.78 & 1.33 & 0.67 & -213.98 & -249.00 & 0.19 \\
\hline TZdEEI 111 & -0.17 & 0.01 & 6.31 & 11.20 & -0.03 & -0.05 & 322.48 & -37.12 & -0.12 \\
\hline TZEEI 13 & 0.13 & 0.03 & -0.97 & -1.91 & 0.02 & 0.19 & -43.97 & -61.52 & -0.10 \\
\hline TZEEI 14 & -0.21 & -0.04 & 0.01 & -2.28 & -0.06 & 0.47 & 193.68 & 104.81 & 0.32 \\
\hline TZEEI 21 & -0.15 & -0.03 & 1.21 & 6.10 & -0.09 & -0.51 & 29.86 & -79.76 & -0.21 \\
\hline TZEEI 29 & 0.23 & 0.04 & -0.10 & -1.88 & 0.13 & -0.15 & -178.00 & 34.70 & -0.01 \\
\hline SE Line & 0.18 & 0.14 & 6.63 & 8.13 & 0.86 & 0.79 & 358.40 & 584.65 & 0.22 \\
\hline SE Tester & 0.18 & 0.03 & 0.77 & 3.51 & 0.08 & 0.37 & 129.22 & 73.36 & 0.19 \\
\hline
\end{tabular}

${ }^{\dagger}$ ASI $=$ Anthesis-silking interval; ${ }^{*},{ }^{* *}$ Significant at $p<0.05$ and $p<0.01$ probability level, respectively. 
SCA effects are associated with dominance and epistatic components of variation, which could be exploited through heterosis breeding. Significant and positive $(p \leq 0.05)$ SCA effects for grain yield were obtained for TZdEEI 54 and TZdEEI 106 in crosses to tester TZEEI 29; TZdEEI 55 and TZdEEI 91 when crossed to TZEEI 14; TZdEEI 84 and TZdEEI 95 when crossed to TZEEI 21 under drought (data not shown), suggesting the importance of epistasis in conferring high yield potential to the hybrids. This also implied that these hybrids could be invaluable in developing superior drought-tolerant three-way hybrids and synthetics. The hybrids TZdEEI $85 \times$ TZEEI 29 and TZdEEI $108 \times$ TZEEI 14 recorded the highest negative SCA effects for ASI under drought and optimal conditions, respectively. Similarly, lines TZdEEI 43, TZdEEI 55 and TZdEEI 62 when crossed to tester TZEEI 29, and lines TZdEEI 45, TZdEEI 91 and TZdEEI 99 when crossed to tester TZEEI 21, had significant $(p<0.05)$ and negative SCA effects for LD (data not shown). This indicated that the hybrids would have a reduced rate of leaf senescence under drought stress, thus prolonging the grain-filling period, which could result in increased grain yield.

\subsection{Phenotypic and Genotypic Correlations for Grain Yield and Other Agronomic Traits of Extra-Early White Maize Hybrids under Drought and Optimal Conditions}

Information on relationships between grain yield and other agronomic traits under drought and optimal conditions would facilitate the identification of appropriate secondary traits for selection for improved grain yield in each research environment. The estimates of the genotypic correlation between grain yield and yield-related traits under managed drought and optimal environments are shown in Tables 6 and 7. The genotypic correlation values were higher than the phenotypic correlation values. Under drought, grain yield had significant but negative phenotypic correlations with DA, DS, ASI, PASP and EASP, whereas positive and significant phenotypic correlations were observed between grain yield and PLHT, EHT and EPP (Table 6). The highest positive phenotypic correlation $\left(r_{p}=0.89\right)$ existed between ASI and DS, whereas the highest negative phenotypic correlation was observed between grain yield and EASP. A strong positive genotypic correlation $\left(\mathrm{r}_{\mathrm{G}}=0.91\right)$ was recorded between grain yield and EPP, whereas a strong negative genotypic correlation $\left(r_{G}=-0.92\right)$ was observed between grain yield and EASP (Table 6). Under optimal growing conditions, EPP, PLHT and EHT had significant and positive genetic correlations with grain yield, whereas significant and negative genetic correlations were obtained between grain yield and DS, DA, ASI, PASP and EASP (Table 7).

Table 6. Phenotypic (above diagonal) and genotypic (below diagonal) correlation coefficients between grain yield and secondary traits ${ }^{\dagger}$ under managed drought at Ikenne during 2013 and 2014 dry seasons.

\begin{tabular}{cccccccccc}
\hline & GY $^{+}$ & DS & DA & ASI & PLHT & EHT & PASP & EASP & EPP \\
\hline GY & - & $-0.41^{* *}$ & $-0.26^{* *}$ & $-0.42^{* *}$ & $0.43^{* *}$ & $0.38^{* *}$ & $-0.74^{* *}$ & $-0.83^{* *}$ & $0.63^{* *}$ \\
DS & $-0.44^{* *}$ & - & $0.89^{* *}$ & $0.60^{* *}$ & $-0.06 \mathrm{~ns}$ & $-0.15^{*}$ & $0.45^{* *}$ & $0.31^{* *}$ & $-0.36^{* *}$ \\
DA & $-0.11^{n s}$ & $0.92^{* *}$ & - & $0.18^{* *}$ & $-0.02 \mathrm{~ns}$ & $-0.09^{* *}$ & $0.33^{* *}$ & $0.15^{*}$ & $-0.26^{* *}$ \\
ASI & $-0.88^{* *}$ & $0.60^{* *}$ & $0.24^{* *}$ & - & $-0.09 \mathrm{~ns}$ & $-0.16 \mathrm{~ns}$ & $0.42^{* *}$ & $0.39^{* *}$ & $-0.32^{* *}$ \\
PLHT & $0.42^{* *}$ & $0.17^{* *}$ & $0.26^{* *}$ & $-0.13^{*}$ & - & $0.49^{* *}$ & $-0.51^{* *}$ & $-0.38^{* *}$ & $0.23^{*}$ \\
EHT & $0.33^{* *}$ & $0.01 \mathrm{~ns}$ & $0.19^{* *}$ & $-0.33^{* *}$ & $0.11 \mathrm{~ns}$ & - & $-0.33^{* *}$ & $-0.34^{* *}$ & $0.20^{* *}$ \\
PASP & $-0.83^{* *}$ & $0.54^{* *}$ & $0.21^{* *}$ & $0.91^{* *}$ & $-0.60^{* *}$ & $-0.06 \mathrm{~ns}$ & - & $0.69^{* *}$ & $-0.50^{* *}$ \\
EASP & $-0.92^{* *}$ & $0.36^{* *}$ & $-0.01_{\mathrm{ns}}$ & $0.89^{* *}$ & $-0.36^{* *}$ & $-0.28^{* *}$ & $0.87^{* *}$ & - & $-0.51^{* *}$ \\
EPP & $0.91^{* *}$ & $-0.54^{* *}$ & $-0.24^{* *}$ & $-0.92^{* *}$ & $0.26^{* *}$ & $0.34^{* *}$ & $-0.72^{* *}$ & $-0.79^{*}$ & - \\
\hline
\end{tabular}

${ }^{*}, * *$ Significant at $p<0.05$ and $p<0.01$ probability level, respectively; $\mathrm{ns}=$ not significant. ${ }^{+} \mathrm{GY}=$ grain yield; DA $=$ Days to anthesis; DS = Days to silking; ASI = Anthesis-silking interval; PLHT = Plant height; PASP = Plant aspect; $\mathrm{EHT}=$ Ear height; $\mathrm{EASP}=$ Ear aspect; $\mathrm{EPP}=$ Ears per plant. 
Table 7. Phenotypic (above diagonal) and genotypic (below diagonal) correlation coefficient between grain yield and secondary traits ${ }^{\dagger}$ under optimal conditions at Ikenne and Bagauda during 2014 cropping season.

\begin{tabular}{cccccccccc}
\hline & GY $^{+}$ & DS & DA & ASI & PLHT & EHT & PASP & EASP & EPP \\
\hline GY & - & $-0.30^{* *}$ & $-0.31^{* *}$ & $-0.05 \mathrm{~ns}$ & $0.38^{* *}$ & $0.37^{* *}$ & $-0.73^{* *}$ & $-0.75^{* *}$ & $0.38^{* *}$ \\
DS & $-0.26^{* *}$ & - & $0.95^{* *}$ & $0.07 \mathrm{~ns}$ & $-0.17^{*}$ & $0.07 \mathrm{~ns}$ & $0.30^{* *}$ & $0.15^{*}$ & $-0.07 \mathrm{~ns}$ \\
DA & $-0.26^{* *}$ & $0.99^{* *}$ & - & $0.23^{* *}$ & $-0.15^{*}$ & $0.08 \mathrm{~ns}$ & $0.32^{* *}$ & $0.18^{*}$ & $-0.06 \mathrm{~ns}$ \\
ASI & $-0.23^{* *}$ & $0.58^{* *}$ & $0.53^{* *}$ & - & $0.09 \mathrm{~ns}$ & $0.11 \mathrm{~ns}$ & $0.09 \mathrm{~ns}$ & $0.03 \mathrm{n}$ & $-0.04 \mathrm{~ns}$ \\
PLHT & $0.44^{* *}$ & $-0.21^{* *}$ & $-0.15^{*}$ & $0.67^{* *}$ & - & $0.63^{* *}$ & $-0.41^{* *}$ & $-0.37^{* *}$ & $0.22^{* *}$ \\
EHT & $0.49^{* *}$ & $0.17^{*}$ & $0.17^{*}$ & $0.41^{* *}$ & $0.69 *$ & - & $-0.34^{* *}$ & $-0.30^{* *}$ & $0.29^{* *}$ \\
PASP & $-0.99^{* *}$ & $0.31^{* *}$ & $0.32^{* *}$ & $0.46^{* *}$ & $-0.52^{* *}$ & $-0.53^{* *}$ & - & $0.60^{* *}$ & $-0.31^{* *}$ \\
EASP & $-0.94^{* *}$ & $0.10 \mathrm{~ns}$ & $0.09 \mathrm{~ns}$ & $0.19^{*}$ & $-0.49^{* *}$ & $-0.44^{* *}$ & $0.92^{* *}$ & - & $-0.21^{*}$ \\
EPP & $0.63^{* *}$ & $-0.06 \mathrm{~ns}$ & $-0.04 \mathrm{~ns}$ & $-0.28^{* *}$ & $0.37^{* *}$ & $0.50^{* *}$ & $-0.84^{* *}$ & -0.60 & - \\
\hline
\end{tabular}

\section{Discussion}

The preponderance of GCA variances over SCA for grain yield DA, DS, ASI, PASP and EASP under drought and optimal conditions implied that additive gene action largely controlled the inheritance of these traits. The implication is that the yield of the maize hybrids under moisture deficit could be enhanced through recurrent selection methods, such as the $S_{1}$ family and the full-sib family selection, and that inbred lines tolerant to drought with high GCA effects could be extracted from improved cycles of selection of derived populations for hybrid development [37]. Contrary to the findings of the present study, Njeri et al. [38] and Umar et al. [39] reported dominance or non-additive gene effects for grain yield over additive effects under managed drought stress. The differences in the results of the two studies could be attributed to the differences in the genetic materials used as well as the differences in the intensity of stress factors in the environments under which the studies were conducted. Furthermore, the existence of additive gene action for grain yield and LD in the present study implied that progress had been made in developing drought-tolerant maize hybrids with genes from Z. diploperennis. The preponderance of SCA over GCA observed for ASI, EPP and LD under managed drought, and ASI, EPP, PASP and EASP under optimal growing conditions suggested that non-additive gene action controlled the expression of these traits. This result indicated that substantial genetic enhancement could also be achieved by employing breeding schemes that capitalize on non-additive gene action, such as hybridization and pedigree selection. Significant GCA $A_{\text {Line }} \times$ location interaction mean squares were obtained for grain yield, DA, DS PLHT and EASP under drought as well as for grain yield, DA, DS, PLHT, EHT, EASP and PASP under optimal growing conditions. Similarly, $\mathrm{GCA}_{\text {Tester }} \times$ location interaction mean squares were significant for grain yield, ASI, PLHT, PASP EASP and LD under managed drought and for grain yield, DA, DS, PLHT, EPP, PASP and EASP under optimal conditions. These results signified variations in the GCA of the parental lines for these traits in different environments. The lack of significant SCA $\times$ location interaction mean squares for grain yield, DA, DS, ASI, PLHT, EHT, EPP, PASP and EASP under drought and optimal conditions indicated that the hybrids were consistent in the expression of the traits in the contrasting environments.

The GCA effects of inbred lines are important for the improvement of target traits in a population and for the development of synthetic varieties and hybrids ([40]. The significant and negative GCA effects observed for LD for inbred TZdEEI 22 under drought conditions indicated that the rate of leaf senescence of its progenies would slow down under drought and that the favourable alleles for this trait could easily be introgressed into tropical white maize populations for improving the yield performance of hybrids and synthetic varieties. The positive and significant GCA effects observed for DS of the three inbred lines, TZdEEI 72, TZdEEI 83 and TZdEEI 107, as well as the ASI for the 
inbreds TZdEEI 83, TZdEEI 94 and TZdEEI 107, under drought suggested that these lines had a high probability of transferring their characteristics to their progenies and could therefore serve as sources of favourable alleles for genetic enhancement of grain yield of tropical maize germplasm under drought conditions. The positive and significant GCA effects observed for TZdEEI 51 and TZdEEI 91 for grain yield under managed drought stress suggested that these two inbred lines have the potential to be successfully utilized in tropical maize breeding programs to combat drought stress as these lines have a high probability of transmitting drought tolerance alleles to their progenies. High GCA indicates the inherent genetic value of a parent due to the presence of additive genetic effects and is fixable [41]. Therefore, the parental inbreds characterized by high GCA values for traits could produce superior segregants in the $\mathrm{F}_{2}$ and later generations as they can serve as vital sources of beneficial alleles [42]. Furthermore, the presence of high GCA effects for grain yield suggested that continued advancement could be made in selecting for increased grain yield. The negative and significant GCA effects detected for the stay green characteristic of TZdEEI 21 implied that this parental line is likely to transmit genes for delayed leaf senescence to its progenies.

It is hard to explain the reasons for the higher heritability for grain yield under drought stress compared to that of optimal conditions because heritability is normally higher under optimal conditions than under stress. The plausible explanation is that the effects of the environmental factors on the grain yield of the genotypes might have been very minimal, most probably due to uniform management conditions under drought stress, and this might have resulted in reduced environmental variance and hence increased narrow sense heritability. Additionally, this could be interpreted to mean that the inbred lines used in the present study might have displayed high genetic variance for grain yield under drought stress. This result disagrees with the findings of earlier researchers $[16,43]$ who reported lower heritability of grain yield under drought environments compared to optimal environments.

The estimates of narrow sense heritability obtained in the present study for grain yield and other measured traits were higher than those reported by Mhike et al. [44], except for DA and ASI. The higher heritability estimates recorded for grain yield, ASI and DS under drought compared to the optimal conditions could also be partly attributed to the fact that the hybrids evaluated in the present study might have inherited drought-tolerance genes from the parental lines derived from Zea diploperennis. The results of the present study confirmed the findings of earlier researchers that ASI, EPP, EASP and LD were effective secondary traits in selecting for enhanced grain yield under moisture stress, thus justifying their inclusion in the IITA base index for selection for drought tolerance [26].

There were significant associations between grain yield and secondary traits examined in the present study. The implications of these results are that EPP, PASP, EASP and ASI could serve as reliable selection indices for improving grain yield under drought. Furthermore, DS, EHT and PLHT were identified as traits of potential importance for the selection of drought-tolerant extra-early maize genotypes. Badu-Apraku et al. [18], Owusu et al. [45] and Songsri et al. [46] reported that correlations between phenotypic characters of inbred lines assessed under stress conditions were usually reduced because of the presence of genotype $\times$ environment interactions. Therefore, the significant genotype $\times$ environment interactions observed in the present study could have reduced the correlations observed between the phenotypic characters of the inbred lines assessed under stress conditions.

\section{Conclusions}

The significant GCA and SCA variances for grain yield and most measured traits in the present study demonstrated that both additive and non-additive genetic effects conditioned the inheritance of these traits; however, additive genetic variances were more important than the non-additive genetic variances. Inbreds TZdEEI 51 and TZdEEI 91 displayed positive and significant GCA effects for grain yield under drought, whereas inbred TZdEEI 22 was outstanding in stay-green characteristic. Ears per plant, plant aspect, ear aspect and anthesis-silking interval were found to be reliable secondary traits for selecting for drought tolerance. Hybrids TZdEEI $54 \times$ TZEEI 13, TZdEEI $91 \times$ TZEEI 21 and TZdEEI $55 \times$ TZEEI 21 were identified as superior in performance under drought stress and should be 
extensively tested in drought-prone environments in SSA and commercialized. The genetic materials developed from Zea diploperennis possessed genes for drought tolerance, with hybrids TZdEEI $54 \times$ TZEEI 13, TZdEEI $91 \times$ TZEEI 21 and TZdEEI $55 \times$ TZEEI 21 displaying high grain yield and drought tolerance imparted by Zea diploperennis. Furthermore, promotion of the superior hybrids identified in the present study would contribute to increased maize production and productivity, enhance farmers' incomes and help in alleviating poverty in SSA.

Author Contributions: Conceptualization, I.K.A. and B.B.-A.; Methodology, I.K.A. and B.B.-A. and software, Formal analysis, I.K.A. and J.T.; Investigation, B.B.-A., G.B.A., and J.A.-D.; resources, B.B.-A.; Data curation, I.K.A., and B.B.-A.; Writing-original draft preparation, I.K.A., J.A.-D.; Writing-review and editing, I.K.A., B.B.-A., J.T., G.B.A., and J.A.-D.; Supervision, B.B.-A.; Project administration, B.B.-A.; Funding acquisition, B.B.-A., and I.K.A. All authors have read and agreed to the published version of the manuscript.

Funding: This work was funded by the Bill and Melinda Gates Foundation [OPP1134248]. The authors are also grateful to the Pan African University for the funding support for this thesis research as well as the technical assistance from the staff of the IITA Maize Improvement Unit.

Acknowledgments: This work was supported by the Bill and Melinda Gates Foundation [OPP1134248]. The authors are grateful for the financial support of the African Union Commission and the International Institute of Tropical Agriculture (IITA), Ibadan. The authors are also grateful for the field assistance of the staff of the Maize Improvement Unit of IITA, Ibadan, Nigeria.

Conflicts of Interest: The authors declare no conflict of interest

\section{References}

1. United States Department of Agriculture (USDA). World Corn Production 2020/2021. 14 May 2020. Available online: http://www.worldagriculturalproduction.com/crops/corn.aspx (accessed on 2 June 2020).

2. Amegbor, I.K.; Badu-Apraku, B.; Annor, B. Combining ability and heterotic patterns of extra-early maturing white maize inbreds with genes from Zea diploperennis under multiple environments. Euphytica 2017, $213,24$. [CrossRef]

3. Edmeades, G.O.; Bolaños, J.; Chapman, S.C.; Lafitte, H.R.; Bänziger, M. Selection improves drought tolerance in tropical maize populations: I. Gains in biomass, grain yield, and harvest index. Crop Sci. 1999, 39, 1306-1315. [CrossRef]

4. Lafitte, H.R.; Edmeades, G.O. Association between traits in tropical maize inbred lines and their hybrids under high and low soil nitrogen. Maydica 1995, 40, 259-267.

5. Rosen, S.; Scott, L. Famine grips sub-Saharan Africa. Out-Look Agric. 1992, 191, $20-24$.

6. Monneveux, P.; Sanchez, C.; Beck, D.; Edmeades, G.O. Drought Tolerance Improvement in Tropical Maize Source Populations: Evidence of Progress. Crop Sci. 2006, 46, 180-191. [CrossRef]

7. Betrán, F.J.; Ribaut, J.M.; Beck, D.; Gonzalez, D.L. Genetic diversity, specific combining ability, and heterosis in tropical maize under stress and nonstress environments. Crop Sci. 2003, 43, 797-806. [CrossRef]

8. Badu-Apraku, B.; Fakorede, M.A.B. Improvement of Early and Extra-Early Maize for Combined Tolerance to Drought and Heat Stress in Sub-Saharan Africa. In Advances in Genetic Enhancement of Early and Extra-Early Maize for Sub-Saharan Africa; Springer: Cham, Switzerland, 2017; pp. 311-358.

9. Du Plessis, J. Maize Production. Directorate of Agricultural Information Services; Department of Agriculture: Pretoria, South Africa, 2003; p. 90.

10. Chathurika, W.; Henry, W.B.; Reddy, K.R. Evaluation of Drought Tolerant Maize Germplasm to Induced Drought Stress. J. Miss. Acad. Sci. 2017, 62, 316-329.

11. Nielsen, R.L. Effects of Stress during Grain Filling in Corn; Corny News Network, Purdue University: West Lafayette, IN, USA, 2018; Available online: https://www.agry.purdue.edu/ext/corn/news/timeless/ grainfillstress.html (accessed on 2 June 2020).

12. Boyle, M.G.; Boyer, J.S.; Morgan, P.W. Stem infusion of liquid culture medium prevents reproductive failure of maize at low water potential. Crop Sci. 1991, 31, 1246-1252. [CrossRef]

13. Edmeades, G.O.; Bolaños, J.; Elings, A.; Ribaut, J.-M.; Bänziger, M.; Westgate, M.E. The Role and Regulation of the Anthesis-Silking Interval in Maize. In Physiology and Modeling Kernel Set in Maize; Westgate, M.E., Boote, K.J., Eds.; CSSA Special Publ. 29; CSSA: Madison, WI, USA, 2000; pp. 43-73. 
14. Grant, R.F.; Jackson, B.S.; Kiniry, J.R.; Arkin, G.F. Water deficit timing effects on yield components in maize. Agron. J. 1989, 81, 61-65. [CrossRef]

15. NeSmith, D.S.; Ritchie, J.T. Effects of soil water-deficits during tassel emergence on development and yield components of maize (Zea mays L.). Field Crops Res. 1992, 28, 251-256. [CrossRef]

16. Bänziger, M.; Cooper, M. Breeding for low input conditions and consequences for participatory plant breeding examples from tropical maize and wheat. Euphytica 2001, 122, 503-519. [CrossRef]

17. Kamara, A.Y.; Menkir, A.; Badu-Apraku, B.; Ibikunle, O. The influence of drought stress on growth, yield and yield components of selected maize genotypes. J. Agric. Sci. 2003, 141, 43-50. [CrossRef]

18. Badu-Apraku, B.; Fakorede, M.A.B.; Oyekunle, M. Selection of extra-early maize inbreds under low N and drought at flowering and grain-filling for hybrid production. Maydica 2011, 56, 36-47.

19. Edmeades, G.O.; Bänziger, M.; Schussler, J.R.; Campos, H. Improving abiotic stress tolerance in maize: A random or planned process? In Proceedings of the Arnel R. Hallauer International Symposium on Plant Breeding, Mexico City, Mexico, 17-22 August 2003; Iowa State University Press: Iowa Ames, IA, USA, 2004.

20. Campos, H.; Cooper, M.; Habben, J.E.; Edmeades, G.O.; Schussler, J.R. Improving drought tolerance in maize: A view from industry. Field Crops Res. 2004, 90, 19-34. [CrossRef]

21. Akaogu, I.C.; Badu-Apraku, B.; Adetimirin, V.O. Combining ability and performance of extra-early maturing yellow maize inbreds in hybrid combinations under drought and rain-fed conditions. J. Agric. Sci. 2017, 155, 1520-1540. [CrossRef]

22. Edmeades, G.O.; Bolaños, J.; Lafitte, H.R. Progress in breeding for drought tolerance in maize. In 47th Annual Corn and Sorghum Industrial Research Conference, Washington, DC, United States of America; Wilkinson, D., Ed.; ASTA: Washington, DC, USA, 1992; pp. 93-111.

23. Gowda, M.; Longin, C.F.H.; Lein, V.; Reif, J.C. Relevance of specific versus general combining ability in winter wheat. Crop Sci. 2012, 52, 2494-2500. [CrossRef]

24. Badu-Apraku, B.; Oyekunle, M. Genetic analysis of grain yield and other traits of extra-early yellow maize inbreds and hybrid performance under contrasting environments. Field Crops Res. 2012, 129, 99-110. [CrossRef]

25. Guei, R.G.; Wassom, C.F. Inheritance of drought adaptive traits in maize. I. Interrelationships between yield, flowering, and ears per plant. Maydica 1992, 37, 157-164.

26. Badu-Apraku, B.; Akinwale, R.O.; Ajala, S.O.; Menkir, A.; Fakorede, M.A.B.; Oyekunle, M. Relationships among traits of tropical early maize cultivars in contrasting environments. Agron. J. 2011, 103, 717-729. [CrossRef]

27. Adebayo, M.A.; Menkir, A.; Blay, E.; Gracen, V.; Danquah, E.; Hearne, S. Genetic analysis of drought tolerance in adapted $x$ exotic crosses of maize inbred lines under managed stress conditions. Euphytica 2014, 196, 261-270. [CrossRef]

28. Ertiro, B.T.; Beyene, Y.; Das, B.; Mugo, S.; Olsen, M.; Oikeh, S.; Juma, C.; Labuschagne, M.; Prasanna, B.M. Combining ability and testcross performance of drought tolerant maize inbred lines under stress and nonstress environments in Kenya. Plant Breed. 2017, 136, 197-205. [CrossRef] [PubMed]

29. Rukundo, P.; Shimelis, H.; Laing, M.; Gahakwa, D. Combining Ability, Maternal Effects, and Heritability of Drought Tolerance, Yield and Yield Components in Sweetpotato. Front. Plant Sci. 2017, 7, 1981. [CrossRef] [PubMed]

30. Menkir, A.; Badu-Apraku, B.; The, C.; Adepoju, A. Evaluation of heterotic patterns of IITA lowland white maize inbred lines. Maydica 2003, 48, 161-170.

31. Oyekunle, M.; Badu-Apraku, B. Genetic analysis of grain yield and other traits of early -maturing maize inbreds under drought and well-watered conditions. J. Agron. Crop Sci. 2014, 200, 92-107. [CrossRef]

32. SAS Institute Inc. Base SAS 9.3 Procedures Guide; SAS Institute Inc.: Cary, NC, USA, 2011.

33. Rodriguez, F.; Alvarado, G.; Pacheco, A.; Burueno, J.; Crossa, J. AGD-R Analysis of Genetic Design in R; International Maize and Wheat Improvement Center: El Batan, Mexico, 2015.

34. Singh, M.; Ceccarelli, S.; Hamblin, J. Estimation of heritability from varietal trials data. Theo. Appl. Genet. 1993, 86, 437-441. [CrossRef]

35. Beck, D.L.; Vasal, S.K.; Crossa, J. Heterosis and combining ability of CIMMYT's tropical early and intermediate maturity maize germplasm. Maydica 1990, 35, 279-285.

36. Baker, R.J. Issues in diallel analysis. Crop Sci. 1978, 18, 533-536. [CrossRef] 
37. Obeng-Bio, E.; Badu-Apraku, B.; Ifie, B.E.; Danquah, A.; Blay, E.T.; Annor, B. Genetic analysis of grain yield and agronomic traits of early provitamin A quality protein maize inbred lines in contrasting environments. $J$. Agric. Sci. 2019, 157, 413-433. [CrossRef]

38. Njeri, S.G.; Makumbi, D.; Warburton, M.L.; Diallo, A.; Jumbo, M.B.; Chemining'wa, G. Genetic analysis of tropical quality protein maize (Zea mays L.) germplasm. Euphytica 2017, 213, 261. [CrossRef]

39. Umar, U.U.; Ado, S.G.; Aba, D.A.; Bugaje, S.M. Estimates of combining ability and gene action in maize (Zea mays L.) under water stress and non-stress conditions. J. Biol. Agric. Healthc. 2014, 4, 25.

40. Akinwale, R.O.; Badu-Apraku, B.; Fakorede, M.A.B.; Vroh-Bi, I. Heterotic grouping of tropical early-maturing maize inbred lines based on combining ability in Striga-infested and Striga-free environments and the use of SSR markers for genotyping. Field Crops Res. 2014, 156, 48-62. [CrossRef]

41. Simmonds, N.W. Principles of Crop Improvement; Longman Group Ltd.: London, UK, 1979; p. 408.

42. Jagadish, N.; Jayalakshmi, V. Combining ability studies for drought tolerance attributes in kabuli chickpea (Cicer arietinum L.). Electron. J. Plant Breed. 2014, 5, 435-441.

43. Shayanowako, A.T.; Laing, M.; Shimelis, H.; Mwadzingeni, L. Resistance breeding and biocontrol of Striga asiatica (L.) Kuntze in maize: A review. Acta Agric. Scand. Sect. B-Plant Soil Sci. 2018, 68, 110-120.

44. Mhike, X.; Lungu, D.M.; Vivek, B. Combining ability studies amongst AREX and CIMMYT maize (Zea mays L.) inbred lines under stress and non-stress conditions. Afr. J. Agric. Res. 2011, 6, 1952-1957.

45. Owusu, E.Y.; Amegbor, I.K.; Darkwa, K.; Oteng-Frimpong, R.; Sie, E.K. Gene action and combining ability studies for grain yield and its related traits in cowpea (Vigna unguiculata). Cogent Food Agric. 2018, 4, 1-17. [CrossRef]

46. Songsri, P.; Jogloy, S.; Kesmala, T.; Vorasoot, N.; Akkasaeng, C.; Patanothai, A.; Holbrook, C.C. Heritability of Drought Resistance Traits and Correlation of Drought Resistance and Agronomic Traits in Peanut. Crop Sci. 2008, 48, 2245-2253. [CrossRef]

(C) 2020 by the authors. Licensee MDPI, Basel, Switzerland. This article is an open access article distributed under the terms and conditions of the Creative Commons Attribution (CC BY) license (http://creativecommons.org/licenses/by/4.0/). 